Brown-HET-1469

\title{
Notes on Collective Field Theory of Matrix and Spin Calogero Models
}

\author{
INÊS ANICETO* and ANTAL JEVICKI ${ }^{\dagger}$ \\ Physics Department \\ Brown University \\ Providence, Rhode Island 02912, USA
}

28th February 2018

\begin{abstract}
Matrix models and related Spin-Calogero-Sutherland models are of major relevance in a variety of subjects, ranging from condensed matter physics to QCD and low dimensional string theory. They are characterized by integrability and exact solvability. Their continuum, field theoretic representations are likewise of definite interest. In this paper we describe various continuum, field theoretic representations of these models based on bosonization and collective field theory techniques. We compare various known representations and describe some nontrivial applications.
\end{abstract}

*email:nes@het.brown.edu

†email:antal@het.brown.edu 


\section{Introduction}

Matrix models in general and specially their simpler N-body cousins can be studied effectively in the large $\mathrm{N}$ limit through continuum, collective field type techniques. The one matrix problem in its fermionic reduction gave a systematic perturbative representation of low dimensional noncritical string theory [1, 2, 3, 4, 5]. Its continuum, collective field representation gave major insight into the origin of the extra (Liouville) dimension and the tachyon mode. On the other hand, understanding of nonperturbative effects such as vortices and black holes clearly requires the full understanding of the theory [6, 7, 8], including possibly gauge degrees of freedom. Recently, for example the dynamics of long strings or FZZT branes was successfully simulated by nonsinglet degrees of the matrix [9]. The continuum, field theoretic representations of these theories can be formulated through techniques of non-abelian bosonization or collective field theory [10, 11, 12, 13]. The resulting hamiltonians are written in conformal field theory notation and exhibit higher, Yangian symmetry of the form studied in [14, 15, 16, 17, 18, 19].

Because of their relevance it is of considerable interest to study further these continuum field theoretic representations. They hold the potential for giving answers to a number of (nonperturbative) problems both in string theory and condensed matter physics.

In this review we describe in some detail the continuum, collective field theory techniques and study the relationship between the continuum field theories that result. We also demonstrate the usefulness of the bosonic, collective field representation by presenting some nontrivial applications: in one we discuss in the field theory setting the linearized spectrum equation, which is shown to take the form of an eigenvalue problem due to Marchesini and Onofri [20]. This eigenvalue problem has recently appeared in number of matrix theory applications. Our second demonstration concerns the treatment of nontrivial backgrounds associated with nontrivial states of the theory. In particular we study the theory in a semiclassical background associated with the quantum many-body state found by Haldane and Ha [21]. This gives a highly nontrivial example where exact results are known and serves as a demonstration of how nontrivial backgrounds appear in the collective field framework.

The outline of the review is as follows. In section 2 we introduce the spin Calogero-Sutherland model and describe in detail its relationship with the matrix model. In section 3 we give details of the bosonization procedure of the spin CS hamiltonian in the conformal field theory language. In section 4 the same hamiltonian is studied using collective fields. Sections 5 and 6 describe some non trivial applications of this theory. Possible, further applications are also discussed in the Conclusion. 


\section{From Matrix Models to Spin Calogero-Sutherland Models}

Various reductions of matrix models lead to simpler many-body problems of Spin CalogeroSutherland type [22, 23, 24, 25, 26, 27, 28, 29, 30, 31, 32, 33, 34, 35, 36, 37, 38. We begin by describing the general scheme for such reductions (in the case of Euclidean metric, the more general case of Riemannian symmetric spaces can be seen in recent work [39, 40]). Consider matrix quantum mechanics defined by the hamiltonian [20, 17]

$$
H=\operatorname{Tr}\left(\frac{1}{2} \Pi^{2}+\left(\frac{\mathrm{N}}{\mathrm{g}}\right) \mathrm{V}\left(\left(\frac{\mathrm{g}}{\mathrm{N}}\right) \Phi^{2}\right)\right)
$$

Here $\Phi$ represents a Hermitian $N \times N$ matrix and the scaling of coupling constants (by $N$ ) in the general, polynomial potential $V$ is done in accordance with 't Hooft's large $N$ limit. The hamiltonian acts on the Hilbert space of square integrable functions defined with respect to the invariant measure $[d \Phi]=\prod \Phi_{a a} \prod_{a<b} d \Phi_{a b} d \Phi_{b a}$.

The invariance of the theory under $S U(N)$ transformations implies that the eigenstates are described by unitary irreducible representations of $S U(N)$. To exhibit the group theoretic degrees of freedom it is convenient to use a "polar" decomposition of the matrix coordinate $\Phi$, and perform a separation of eigenvalues (of the matrix) and the angles

$$
\Phi=\Omega^{\dagger} \Lambda \Omega \quad \Leftrightarrow \quad \Phi_{a b}=\sum_{i}\left(\Omega^{\dagger}\right)_{a i} \lambda_{i}(\Omega)_{i b},
$$

where $\Lambda=\operatorname{diag}\left(\lambda_{1}, \ldots, \lambda_{\mathrm{N}}\right)$. Indices $a, b, c=1, \ldots, N$, and $i, j=1, \ldots, N$, will denote the internal indices throughout. One notes that $\Omega \in S U(N) / \mathbb{H}$, with $\mathbb{H}$ being the stability subgroup of $\Lambda$ under $S U(N)$. Equation (2) can be rewritten as

$$
\Phi_{a b}\left(\Omega^{\dagger}\right)_{b i}=\lambda_{i}\left(\Omega^{\dagger}\right)_{a i}
$$

so we can conclude that $\left(\Omega^{\dagger}\right)_{b i}$ is the component $b$ of the $i$ th eigenvector of the matrix $\Phi$, with corresponding eigenvalue $\lambda_{i}$.

Defining vectors $Z^{i}$ and $\bar{Z}^{i}$ by their components $Z_{a}^{i}=\Omega_{i a}$ and $\bar{Z}_{a}^{i}=\left(\Omega^{\dagger}\right)_{a i}=\bar{\Omega}_{i a}\left(\bar{Z}_{a}^{i}\right.$ is the complex conjugate of $Z_{a}^{i}$ ), we can determine the constraints on these vectors coming from $\Omega \in U(N)$. Because $\Omega$ is unitary, we have

$$
\begin{array}{lll}
\Omega^{\dagger} \Omega=1 & \Rightarrow & \sum_{i} \bar{Z}_{a}^{i} Z_{b}^{i}=\delta_{a b} \\
\Omega \Omega^{\dagger}=1 & \Rightarrow & \sum_{a} Z_{a}^{i} \bar{Z}_{a}^{j}=\delta_{i j} .
\end{array}
$$

These two constraints state the completeness and orthonormality of the eigenfunctions, respec- 
tively. We can write the eigenfunction equation in terms of the eigenvectors $\bar{Z}^{i}$ as:

$$
\Phi_{a b} \bar{Z}_{b}^{i}=\lambda^{i} \bar{Z}_{a}^{i}
$$

To evaluate the form of the hamiltonian after the above transformation one needs the following identities, obtained by using the chain rule:

$$
\begin{aligned}
\frac{\delta \lambda^{i}}{\delta \Phi_{b c}} & =Z_{b}^{i} \bar{Z}_{c}^{i}, \\
\frac{\delta \bar{Z}_{a}^{i}}{\delta \Phi_{b c}} & =\sum_{j(\neq i)} \frac{\bar{Z}_{a}^{j} Z_{b}^{j} \bar{Z}_{c}^{i}}{\lambda_{i}-\lambda_{j}} \\
\frac{\delta Z_{a}^{i}}{\delta \Phi_{b c}} & =\sum_{j(\neq i)} \frac{Z_{b}^{i} \bar{Z}_{c}^{j} Z_{a}^{j}}{\lambda_{i}-\lambda_{j}} .
\end{aligned}
$$

We can now apply these rules to the kinetic term (we will use the notation where repeated indices are summed over, unless stated otherwise). First:

$$
\frac{\partial}{\partial \Phi_{b c}}=Z_{b}^{i} \bar{Z}_{c}^{i} \frac{\partial}{\partial \lambda_{i}}+\sum_{j(\neq i)} \frac{Z_{b}^{i} \bar{Z}_{c}^{j} Z_{a}^{j}}{\lambda_{i}-\lambda_{j}} \frac{\partial}{\partial Z_{a}^{i}}+\sum_{j(\neq i)} \frac{\bar{Z}_{a}^{j} Z_{b}^{j} \bar{Z}_{c}^{i}}{\lambda_{i}-\lambda_{j}} \frac{\partial}{\partial \bar{Z}_{a}^{i}},
$$

and then:

$$
-\operatorname{Tr}\left(\Pi^{2}\right)=\sum_{b, c} \frac{\partial}{\partial \Phi_{c b}} \frac{\partial}{\partial \Phi_{b c}}=\sum_{i} \frac{\partial^{2}}{\partial \lambda_{i}^{2}}+2 \sum_{i \neq j} \frac{1}{\lambda_{i}-\lambda_{j}} \frac{\partial}{\partial \lambda_{i}}+\sum_{i \neq j} \frac{\bar{Q}_{i j} Q_{i j}}{\left(\lambda_{i}-\lambda_{j}\right)^{2}},
$$

where we defined

$$
\begin{aligned}
Q_{i j} & \equiv i \sum_{a}\left\{Z_{a}^{i} \frac{\partial}{\partial Z_{a}^{j}}-\bar{Z}_{a}^{j} \frac{\partial}{\partial \bar{Z}_{a}^{i}}\right\} \equiv i\left\{Z^{i} \frac{\partial}{\partial Z^{j}}-\bar{Z}^{j} \frac{\partial}{\partial \bar{Z}^{i}}\right\}, \\
\bar{Q}_{i j} & =Q_{j i}=i\left\{Z^{j} \frac{\partial}{\partial Z^{i}}-\bar{Z}^{i} \frac{\partial}{\partial \bar{Z}^{j}}\right\} .
\end{aligned}
$$

The sum in the matrix variable $a$ has been omitted. The $Q_{i j}$ is a set of noncommuting differential operators that only depend on the angular coordinates. An equivalent way of writing the result above is [20]:

$$
\operatorname{Tr}\left(\Pi^{2}\right)=-\frac{1}{\Delta} \sum_{\mathrm{i}} \frac{\partial^{2}}{\partial \lambda_{\mathrm{i}}^{2}} \Delta-\sum_{\mathrm{i} \neq \mathrm{j}} \frac{\mathrm{Q}_{\mathrm{ij}} \mathrm{Q}_{\mathrm{ji}}}{\left(\lambda_{\mathrm{i}}-\lambda_{\mathrm{j}}\right)^{2}} .
$$

Here $\Delta=\prod_{i<j}\left(\lambda_{i}-\lambda_{j}\right)$ is the Vandermonde determinant, representing the invariant measure $[d \Phi]=\Delta^{2} d \lambda_{1} \cdots d \lambda_{N}[d \Omega]$, where $[d \Omega]$ is the invariant volume element in $U(N)$. An equivalent way of deriving (8), based on the line element, can be found for example in [20]. In general, the potential terms are easily expressible in terms of the eigenvalues and the full Matrix hamiltonian 
becomes

$$
H=-\frac{1}{2} \frac{1}{\Delta} \sum_{i} \frac{\partial^{2}}{\partial \lambda_{i}^{2}} \Delta+\frac{N}{g} \sum_{i} V\left(\left(\frac{g}{N}\right) \lambda_{i}^{2}\right)+\sum_{i \neq j} \frac{Q_{i j}^{\mathcal{R}} Q_{j i}^{\mathcal{R}}}{\left(\lambda_{i}-\lambda_{j}\right)^{2}}
$$

We have put in this last result the explicit dependence on the representation $\mathcal{R}$ of $S U(N)$, as the $Q_{i j}^{\mathcal{R}}$ are the matrices corresponding to the $i j$ generator of $S U(N)$ in the representation $\mathcal{R}$ [9, 17, since the theory can be studied in each sector separately. ${ }^{1}$ In the simplest case of $S U(N)$ invariant singlet states the matrix model reduces to a many-body problem of free fermions. The system with $V$ being an inverted oscillator represents 2D non-critical string theory. Classical hamiltonian reduction with a particular (non zero) value of angular momentum is known to lead to a Calogero-type hamiltonian for the eigenvalues [41, 42]. In order to obtain a trigonometric form of the hamiltonian, namely the Calogero-Sutherland Model, one uses unitary matrix model (with $\Phi$ unitary). Continuing our discussion at the quantum level the next relevant case is that of adjoint states of $S U(N)$, studied first in [20] and a number of works thereafter.

Models of similar type also show up in the singlet sector of matrix-vector type theories [12]. We have in the above introduced a notation where the eigenvectors of the matrix appear as vectorlike variables to emphasize the correspondence with that type of theories. In the full matrix theory case the flavour index a clearly ranges from 1 to $\mathrm{N}$. One can then consider a reduction in the number of vector degrees of freedom to a fixed number $\mathrm{R}$. The particular case of a spin chain appears if we represent the operators of the $\mathrm{SU}(\mathrm{N})$ algebra through fermions $Q_{i j}=\sum_{\alpha=1}^{R} \Psi_{\alpha}^{\dagger}(i) \Psi_{\alpha}(j)$, and constrain the value of the number operator at each site

$$
\mathcal{N}_{i} \equiv \sum_{\alpha=1}^{R} \Psi_{\alpha}^{\dagger}(i) \Psi_{\alpha}(i)=1
$$

Here $\Psi_{\alpha}(i)(\alpha=1, \ldots, R)$ are complex $U(N)$-vector fermionic degrees of freedom, and $R$ labels the number of independent components acting like a flavour index [22, 23].

With the above constraint we can write

$$
Q_{i j} Q_{j i}=\left(1-\sum_{\alpha, \beta=1}^{R} Q_{\alpha \beta}(i) Q_{\beta \alpha}(j)\right)
$$

by defining $Q_{\alpha \beta}(i) \equiv \Psi_{\alpha}^{\dagger}(i) \Psi_{\beta}(i)$, and obtain a representation with generators at each site:

$$
Q_{i j} Q_{j i}=\left(1-J_{i} \cdot J_{j}\right)
$$

This corresponds to a Haldane-Shastry spin-spin interaction [25, 26, 27, 21].

\footnotetext{
${ }^{1}$ One further comment is in place. The possible representations $\mathcal{R}$ of $S U(N)$ are restricted to irreducible representations which have a state with all weights equal to zero [6] 9 .
} 
Our main concern in the present review is the continuum, field theoretic representation of this class of models. This representation is based on composite, collective fields and can be reached through various bosonization procedures. The basic form of such continuum, field theoretic representation of the many-body hamiltonian is given by a conformal field theory with dynamical $S U(R)$ charges [12]

$$
\begin{aligned}
H= & b \oint z^{2}\left(\frac{1}{6}(\alpha(z))^{3}+\alpha(z) T^{J}(z)\right)+c(R) W(z) \\
& +\oint d z \oint d w \frac{z w}{(z-w)^{2}}(J(z) \cdot J(w)+\alpha(z) \alpha(w)) .
\end{aligned}
$$

In the above hamiltonian $b$ is a general constant and $c(R)$ is a specific $R$-dependent constant. $\alpha(z)$ is a bosonic scalar field coupled to a level $k=1$ current algebra, which participates in the hamiltonian through a spin-2 $W$-algebra energy-momentum tensor $T^{J}(z)$ and a spin-3 $W$ algebra generator $W(z)$, both built from the $s u(R)$ current algebra [43. One should be aware of the fact that the full hamiltonian will have both $k=1$ and $k=-1$ current algebras, and the corresponding bosonic $U(1)$ scalar fields .

For the purposes of this paper we will concern ourselves with the particular case of $s u(2)$, namely $R=2$, which will be used to illustrate most of the relevant dynamical features. More details of the general case of $s u(R)$ and also the large $R$ limit can be found in [12] and [44. Of considerable future interest is the case $R=N$ with $N \rightarrow \infty$. Aspects of the $R=N \rightarrow \infty$ theory were already given in [44]. Through the large $N$ limit of the current algebra one realizes a $W_{\infty}$ algebra, the expected symmetry of the matrix model [45].

\section{Current-Algebra Representation}

We will now proceed with the study of the Spin Calogero-Sutherland model by describing in detail its bosonized current algebra representation. The standard form of the Spin Calogero-Sutherland model is given by (for example, [21]):

$$
H=-\frac{1}{2} \sum_{i} \frac{\partial^{2}}{\partial x_{i}^{2}}+\frac{1}{4}\left(\frac{\pi}{L}\right)^{2} \sum_{i \neq j} \frac{\beta\left(2 \beta+1+\vec{\sigma}_{i} \cdot \vec{\sigma}_{j}\right)}{\sin ^{2}\left[\frac{\pi}{L}\left(x_{i}-x_{j}\right)\right]}
$$

where $\frac{2 \pi}{L} x_{i} \in[0,2 \pi]$ is the coordinate on the circle.

The spin-spin interaction is equivalently written in terms of the spin exchange operator $P_{i j}$. Recalling that $J_{i}^{a}=\frac{1}{2} \sigma_{i}^{a}$ for each particle $i$, we have the relation:

$$
P_{i j}=J_{i}^{+} J_{j}^{-}+J_{i}^{-} J_{j}^{+}+2\left(J_{i}^{z} J_{j}^{z}+\frac{1}{4}\right)=\frac{1}{2}\left[\vec{\sigma}_{i} \cdot \vec{\sigma}_{j}+1\right] .
$$


Consequently, we can rewrite (11) as:

$$
H=\left(\frac{2 \pi}{L}\right)^{2} \frac{1}{2}\left\{\sum_{i} D_{i}^{2}-\sum_{i \neq j} \frac{z_{i} z_{j}}{\left(z_{i}-z_{j}\right)^{2}} \beta\left(\beta+P_{i j}\right)\right\}
$$

where $D_{i}=z_{i} \frac{\partial}{\partial z_{i}}$, and $z_{i}=e^{i \frac{2 \pi}{L} x_{i}}$. From now on, we will drop the factor of $\left(\frac{2 \pi}{L}\right)$ from the hamiltonian. In general, models of Calogero-type possess several universal properties. First of all they describe particles with generalized statistics, governed by the coupling constant $\beta$, see [46]. Introducing a position exchange operator $K_{i j}$, we have that $P_{i j} K_{i j}=2 x-1(x=+1$ is the case of boson, while the $x=0$ is for fermions), for the corresponding wavefunctions

$$
\Psi\left(\ldots, z_{i} \sigma_{i}, \ldots, z_{j} \sigma_{j}, \ldots\right)=(-1)^{x+1} \Psi\left(\ldots, z_{j} \sigma_{j}, \ldots, z_{i} \sigma_{i}, \ldots\right)
$$

For the bosonization method we will have to consider a system of fermions. In general one can go to a fermionic (or bosonic) versions of the hamiltonian by applying a similarity transformation

$$
\tilde{H}=2\left(\frac{L}{2 \pi}\right)^{2} \Delta^{-\beta} H \Delta^{\beta}-E_{0},
$$

where

$$
\begin{aligned}
\Delta^{\beta} & =\prod_{i<j} \sin ^{\beta}\left(\frac{1}{2}\left(x_{i}-x_{j}\right)\right)=\prod_{i<j}\left(z_{i}-z_{j}\right)^{\beta} \prod_{i} z_{i}^{-\beta \frac{N-1}{2}} \\
E_{0} & =\beta^{2} \frac{N\left(N^{2}-1\right)}{12}
\end{aligned}
$$

By means of some identities, such as

$$
\frac{z_{i} z_{k}}{\left(z_{j}-z_{i}\right)\left(z_{j}-z_{k}\right)}+\frac{z_{k} z_{j}}{\left(z_{i}-z_{k}\right)\left(z_{i}-z_{j}\right)}+\frac{z_{j} z_{i}}{\left(z_{k}-z_{j}\right)\left(z_{k}-z_{i}\right)}=1
$$

the similarity transformation given above provides an effective fermionic hamiltonian of the form

$$
\tilde{H}=\sum_{i} D_{i}^{2}+\frac{\beta}{2} \sum_{i \neq j} \frac{z_{i}+z_{j}}{z_{i}-z_{j}}\left(D_{i}-D_{j}\right)-\frac{\beta}{2} \sum_{i \neq j} \frac{z_{i} z_{j}}{\left(z_{i}-z_{j}\right)^{2}}\left(3+\vec{\sigma}_{i} \cdot \vec{\sigma}_{j}\right) .
$$

Through standard second quantization, the hamiltonian (13) is written in terms of fermionic fields

$$
\widetilde{\Psi}=\left(\begin{array}{l}
\psi_{1} \\
\psi_{2}
\end{array}\right) \quad, \quad J^{i}=\widetilde{\Psi}^{\dagger} \frac{\sigma^{i}}{2} \widetilde{\Psi}(i=1,2,3)
$$


obeying the anti-commutation relations $(a=1,2)$ :

$$
\left\{\psi_{a}(x), \psi_{b}(y)\right\}=\delta_{a b} \delta(x-y) .
$$

Before continuing, we shall review some important aspects of the bosonization procedure.

In the abelian bosonization procedure, each fermi field $\psi, \psi^{\dagger}$ is expressed in terms of a boson $\phi$ by the formulas (as references on bosonization, see [43, 47, 48]) :

$$
\psi(\theta)=\frac{1}{\sqrt{2 \pi}}: e^{-i \phi(z)}: \quad, \quad \psi^{\dagger}(\theta)=\frac{z}{\sqrt{2 \pi}}: e^{i \phi(z)}:=z \psi^{\dagger}(z) .
$$

As there are two fermion fields $\psi_{a}$, two bosons $\phi_{a}$ will be needed. Because we have more than one species of fermionic fields (i.e. two), we also need to employ Klein factors to ensure the anticommutativity among the species. This will be particularly relevant when considering the 4 -fermion interactions later.

After normal ordering one has:

$$
\begin{aligned}
: \psi^{\dagger}\left(w^{\prime}\right) \psi(w): & =\frac{i w}{2 \pi} \partial_{w} \phi \\
: \psi^{\dagger}\left(w^{\prime}\right) \partial_{w} \psi(w): & =\frac{i w}{2 \pi}\left\{\frac{i}{2} \partial_{w}^{2} \phi+\frac{1}{2}\left(\partial_{w} \phi\right)^{2}\right\} \\
: \psi^{\dagger}\left(w^{\prime}\right) \partial_{w}^{2} \psi(w): & =\frac{w}{2 \pi}\left\{\frac{i}{3} \partial_{w}^{3} \phi+\left(\partial_{w} \phi\right)\left(\partial_{w}^{2} \phi\right)-\frac{i}{3}\left(\partial_{w} \phi\right)^{3}\right\} .
\end{aligned}
$$

For the $U(1)$ currents $\alpha^{a}(z),(a=1,2)$ associated with each fermionic field $\psi_{a}(z)$ we have

$$
\alpha^{a}(z) \equiv \psi_{a}^{\dagger}(z) \psi_{a}(z)=i \partial_{z} \phi_{a}
$$

For each of the fermions, the energy-momentum tensor is of the form

$$
T^{a}=-\psi_{a}^{\dagger} \partial \psi_{a}=\frac{1}{2}\left(\partial \psi_{a}^{\dagger} \psi_{a}-\psi_{a}^{\dagger} \partial \psi_{a}\right)-\frac{1}{2} \partial\left(\psi_{a}^{\dagger} \psi_{a}\right)
$$

corresponding to a central charge $c=-2 .^{2}$

A central role will be played by the $S U(2)$ currents given by

$$
\begin{aligned}
& J^{z}=\frac{1}{2}\left[\psi_{1}^{\dagger} \psi_{1}-\psi_{2}^{\dagger} \psi_{2}\right]=\frac{1}{2} J^{1}(z)-\frac{1}{2} J^{2}(z) \\
& J^{ \pm}=\frac{1}{2} \widetilde{\Psi}^{\dagger}\left(\sigma^{1} \pm i \sigma^{2}\right) \widetilde{\Psi} \Rightarrow J^{+}=\psi_{1}^{\dagger} \psi_{2}, \quad J^{-}=\psi_{2}^{\dagger} \psi_{1} .
\end{aligned}
$$

\footnotetext{
${ }^{2}$ For an energy-momentum tensor of the form $T=\frac{1}{2}\left(\partial \psi^{\dagger} \psi-\psi^{\dagger} \partial \psi\right)+\mu \partial\left(\psi^{\dagger} \psi\right)$ the central charge equals $c=1-12 \mu^{2}$.
} 
They obey a level-1 su(2) current algebra with the energy momentum tensor being

$$
T(z)=-\widetilde{\Psi}^{\dagger} \partial \widetilde{\Psi}=-\psi_{1}^{\dagger} \partial \psi_{1}-\psi_{2}^{\dagger} \partial \psi_{2}=T^{1}(z)+T^{2}(z) .
$$

This will generate a Virasoro algebra of central charge $c=-4$, which is expected as we are looking at a theory with two anticommuting fermions $\psi^{1}, \psi^{2}$.

We can proceed with the bosonization of the hamiltonian. We distinguish three distinct terms in the hamiltonian as:

$$
\tilde{H}=\underbrace{-\sum_{i=1}^{N} \frac{\partial^{2}}{\partial x_{i}^{2}}}_{H_{1}} \underbrace{-\frac{\beta}{2} \sum_{i \neq j} \cot \left(\frac{x_{i}-x_{j}}{2}\right)\left(\frac{\partial}{\partial x_{i}}-\frac{\partial}{\partial x_{j}}\right)}_{H_{2}}+\underbrace{\frac{\beta}{4} \sum_{i \neq j} \sin ^{-2}\left(\frac{x_{i}-x_{j}}{2}\right)\left(1+P_{i j}\right)}_{H_{s}} .
$$

The first term in the hamiltonian becomes:

$$
\begin{aligned}
H_{1} & \rightarrow-\int d x \widetilde{\Psi}^{\dagger}(x) \frac{\partial^{2}}{\partial x^{2}} \widetilde{\Psi}(x)=\oint \frac{d z}{i z} \widetilde{\Psi}^{\dagger}(z)\left[z \partial+z^{2} \partial^{2}\right] \widetilde{\Psi}(z) \\
& =\sum_{a} \oint \frac{d z}{2 \pi i}\left[z^{2}\left\{\frac{i}{3} \partial^{3} \phi_{a}+\partial \phi_{a} \partial^{2} \phi_{a}-\frac{i}{3}\left(\partial \phi_{a}\right)^{3}\right\}+z\left\{\frac{i}{2} \partial^{2} \phi_{a}+\frac{1}{2}\left(\partial \phi_{a}\right)^{2}\right\}\right] .
\end{aligned}
$$

In the case of the second term, we have to be more careful since one has a pole at coincident points (this we handle by point-splitting regularization):

$$
H_{2} \rightarrow-\beta \int d x \int d y \widetilde{\Psi}^{\dagger}(x) \widetilde{\Psi}^{\dagger}(y) \cot \left(\frac{x-y}{2}\right) \widetilde{\Psi}(y) \frac{\partial}{\partial x} \widetilde{\Psi}(x)+\beta \int d x \widetilde{\Psi}^{\dagger}(x) \frac{\partial^{2}}{\partial x^{2}} \widetilde{\Psi}(x) .
$$

Note that the last term is just $H_{1}$, bosonized before. Going to complex coordinates,

$$
\begin{aligned}
H_{2} & =-\beta \sum_{a, b} \oint \frac{d z}{z} \oint \frac{d w}{w} \frac{z+w}{z-w} \psi_{a}^{\dagger}(z) \psi_{b}^{\dagger}(w) \psi_{b}(w) z \partial_{z} \psi_{a}(z)-\beta H_{1} \\
& =\beta \sum_{a, b} \oint \frac{d z}{2 \pi i} \oint \frac{d w}{2 \pi i}\left(\frac{z+w}{z-w}\right) i \partial_{w} \phi_{b} z\left[\frac{i}{2} \partial_{z}^{2} \phi_{a}+\frac{1}{2}\left(\partial_{z} \phi_{a}\right)^{2}\right]-\beta H_{1}
\end{aligned}
$$

where the contour integral of $w$ has to be averaged over an outer and an inner circles around the $z$ contour. 
Finally we come to the bosonization of the last term $H_{s}$ of our Hamiltonian:

$$
\begin{aligned}
& H_{s}=\frac{\beta}{8} \sum_{i \neq j} \sin ^{-2}\left(\frac{x_{i}-x_{j}}{2}\right)\left(3+\vec{\sigma}_{i} \cdot \vec{\sigma}_{j}\right) \\
& \rightarrow \frac{3 \beta}{8} \int d x \int d y \widetilde{\Psi}^{\dagger}(x) \widetilde{\Psi}^{\dagger}(y) \sin ^{-2}\left(\frac{x-y}{2}\right) \widetilde{\Psi}(y) \widetilde{\Psi}(x)+ \\
& \quad+\frac{\beta}{8} \sum_{k=1}^{3} \int d x \int d y \sin ^{-2}\left(\frac{x-y}{2}\right) \widetilde{\Psi}^{\dagger}(x) \widetilde{\Psi}^{\dagger}(y) \sigma^{k} \widetilde{\Psi}(y) \sigma^{k} \widetilde{\Psi}(x) .
\end{aligned}
$$

Again we encounter a singularity, the pole is now of second order, and so a regularization gives a zero contribution. Going to the complex plane,

$$
H_{s}=-\frac{3 \beta}{2} \sum_{a, b} \oint \frac{d z}{2 \pi i} \oint \frac{d w}{2 \pi i} \frac{i \partial_{z} \phi_{a} z w i \partial_{w} \phi_{b}}{(z-w)^{2}}+\frac{\beta}{2} \sum_{k} \int d x \int d y \frac{\widetilde{\Psi}^{\dagger}(y) \frac{\sigma^{k}}{2} \widetilde{\Psi}(y) \widetilde{\Psi}^{\dagger}(x) \frac{\sigma^{k}}{2} \widetilde{\Psi}(x)}{\sin ^{2}\left(\frac{x-y}{2}\right)},
$$

where the integration on $w$ is to be done around the $z$ contour. Important to point out is the bosonized representation for the spin permutation operator $P_{i j}$ :

$$
\begin{aligned}
-\beta \sum_{i \neq j} \frac{z_{i} z_{j}}{\left(z_{i}-z_{j}\right)^{2}} P_{i j} & \rightarrow \beta \sum_{a, b} \oint d z \oint d w \frac{\psi_{b}^{\dagger}(z) \psi_{a}^{\dagger}(w) \psi_{b}(w) \psi_{a}(z)}{(z-w)^{2}} \\
& =\beta \sum_{a, b} \oint \frac{d z}{2 \pi i} \oint \frac{d w}{2 \pi i}: e^{-i\left(\phi_{a}(w)-\phi_{b}(w)\right)}: \frac{z w}{(z-w)^{2}}: e^{-i\left(\phi_{b}(z)-\phi_{a}(z)\right)}:,
\end{aligned}
$$

because it has a very similar form to the one that will be found in the next section by collective field theory methods.

Returning to the full hamiltonian, its final form in the bosonized representation reads:

$$
\begin{aligned}
\tilde{H}= & \frac{1-\beta}{6} \oint \frac{d z}{2 \pi i}\left[2 z^{2} \alpha_{a}^{3}+3 z \alpha_{a}^{2}+\alpha_{a}\right]+\frac{\beta}{2} \oint \frac{d z}{2 \pi i} \oint \frac{d w}{2 \pi i} \frac{z+w}{z-w} \alpha_{b}(w) z\left[\partial \alpha_{a}(z)-z \alpha_{a}^{2}(z)\right] \\
& -\frac{3 \beta}{2} \oint \frac{d z}{2 \pi i} \oint \frac{d w}{2 \pi i} \frac{z w}{(z-w)^{2}} \alpha_{a}(z) \alpha_{b}(w)+2 \beta \oint d z \oint d w \frac{z w}{(z-w)^{2}} \sum_{k=1}^{3} J^{k}(w) J^{k}(z) .
\end{aligned}
$$

The indices $a, b$ are summed over, and $\alpha_{a}=i \partial \phi_{a}$. We can also express the Hamiltonian in terms of the spin $\beta$ and charge $B$ bosons, ${ }^{3}$ given by:

$$
B(x)=\alpha_{1}(x)+\alpha_{2}(x) \quad ; \quad \beta(x)=\alpha_{1}(x)-\alpha_{2}(x) .
$$

\footnotetext{
${ }^{3}$ We trust the reader will be able to distinguish between the statistics parameter and the spin field, both labeled $\beta$. The latter will be supplemented with an argument, for example $\beta(z)$, in order to avoid confusion.
} 
With these we have:

$$
\begin{aligned}
\tilde{H}= & \frac{1-\beta}{12} \oint \frac{d z}{2 \pi i}\left[z^{2} B(z)\left(B^{2}(z)+3 \beta^{2}(z)\right)+3 z\left(B^{2}(z)+\beta^{2}(z)\right)+2 B(z)\right]- \\
& +\frac{\beta}{2} \oint \frac{d z}{2 \pi i} \oint \frac{d w}{2 \pi i}\left(\frac{z+w}{z-w}\right) B(w)\left[z \partial B(z)-\frac{1}{2} z\left(B^{2}(z)+\beta^{2}(z)\right)\right]+ \\
& -\frac{3 \beta}{2} \oint \frac{d z}{2 \pi i} \oint \frac{d w}{2 \pi i} \frac{z w}{(z-w)^{2}} B(z) B(w)+2 \beta \oint d z \oint d w \frac{z w}{(z-w)^{2}} \sum_{k=1}^{3} J^{k}(w) J^{k}(z) .
\end{aligned}
$$

We can see in this final expression the general structure given in equation (10). We will discuss in more detail a comparison of various representation further below. We will furthermore see that the same form of the hamiltonian can be obtained by collective field techniques as a second way of determining the bosonized hamiltonian. Finally, the usefulness of the bosonized representation will be demonstrated in selected applications involving studies of nontrivial classical background.

\subsection{Mode Expansion}

It is useful to present also the mode expansion of the bosonized Hamiltonian. We start by introducing the mode expansions for the fermionic fields $\psi_{a}(a=1,2)$ :

$$
\psi_{a}^{\dagger}(z)=\sum_{n} \psi_{-n}^{a \dagger} z^{-n-1} \quad, \quad \psi_{a}(z)=\sum_{n} \psi_{n}^{a} z^{-n}
$$

with commutation relations $\left\{\psi_{n}^{a}, \psi_{m}^{b \dagger}\right\}=\delta_{m, n} \delta^{a b}$. We also need the expansion of the $U(1)$ currents:

$$
\alpha_{a}(z) \equiv \psi_{a}^{\dagger}(z) \psi_{a}(z)=i \partial_{z} \phi_{a}=\sum_{n} \alpha_{n}^{a} z^{-n-1}
$$

with commutation relations $\left[\alpha_{n}^{a}, \alpha_{m}^{b}\right]=n \delta^{a b} \delta_{m+n, 0}$. The components of the current $\alpha^{a}$ can be written in terms of the fermionic field modes as:

$$
\alpha_{n}^{a}=\oint \frac{d z}{2 \pi i} z^{n} \psi_{a}^{\dagger}(z) \psi_{a}(z)=\sum_{\ell, m} \oint \frac{d z}{2 \pi i} z^{n-(\ell+m)-1} \psi_{-\ell}^{a \dagger} \psi_{m}^{a}=\sum_{m} \psi_{m-n}^{a \dagger} \psi_{m}^{a} .
$$

For each of the fermions we have the energy-momentum tensor,

$$
T^{a}=-\psi_{a}^{\dagger} \partial \psi_{a}=\sum_{n} L_{n}^{a} z^{-n-2}
$$

The Virasoro generators $L_{n}^{a}$ are given by:

$$
L_{n}^{a}=-\frac{1}{2} \oint \frac{d z}{2 \pi i} z^{n+1} \psi_{a}^{\dagger} \partial \psi_{a}=-\sum_{\ell, m} \oint \frac{d z}{2 \pi i} z^{n-(\ell+m)-1}(-m) \psi_{-\ell}^{a \dagger} \psi_{m}^{a}=\sum_{m} m \psi_{m-n}^{a \dagger} \psi_{m}^{a} .
$$


Consider next the operators of a $s u(2)$ current algebra $J^{i}(z)(i, j, k=1,2,3 \equiv x, y, z)$. These can be expanded as $J^{i}(z)=\frac{1}{2 \pi} \sum_{N} J_{N}^{i} z^{-N-1}$, with

$$
\begin{aligned}
2 \pi J_{n}^{x} & =\frac{1}{2}\left(J^{+}+J^{-}\right)_{n}=\frac{1}{2} \sum_{m}\left[\psi_{(m-n)}^{1 \dagger} \psi_{m}^{2}+\psi_{(m-n)}^{2 \dagger} \psi_{m}^{1}\right] \\
2 \pi J_{n}^{y} & =\frac{1}{2 i}\left(J^{+}-J^{-}\right)_{n}=-\frac{i}{2} \sum_{m}\left[\psi_{(m-n)}^{1 \dagger} \psi_{m}^{2}-\psi_{(m-n)}^{2 \dagger} \psi_{m}^{1}\right] \\
2 \pi J_{n}^{z} & =\frac{1}{2}\left(\alpha_{n}^{1}-\alpha_{n}^{2}\right)=-\frac{1}{2} \sum_{m}\left[\Psi_{(m-n)}^{2 \dagger} \psi_{m}^{2}-\psi_{(m-n)}^{1 \dagger} \psi_{m}^{1}\right]
\end{aligned}
$$

with the commutation relations

$$
\left[J_{n}^{i}, J_{m}^{j}\right]=i \varepsilon^{i j k} J_{m+n}^{k}+\frac{n}{2} \delta^{i j} \delta_{m+n, 0}
$$

We already introduced the charge field (the $B$ field) and the spin field (the $\beta$ field), and saw that to bosonize the hamiltonian, we needed two scalar bosonic fields $\alpha_{1}, \alpha_{2}$, because we were interested in the $S U(2)$ current algebra. If we now identify $\alpha_{i} \equiv \alpha^{\sigma_{i}}$, where $\sigma= \pm 1$ (or alternatively $\sigma_{1}=\uparrow$ and $\left.\sigma_{2}=\downarrow\right)$, then the following expansions hold, in terms of $z$ coordinates:

$$
\left\{\begin{array} { l } 
{ B ( z ) = \sum _ { n } B _ { n } z ^ { - n - 1 } } \\
{ \beta ( z ) = \sum _ { n } \beta _ { n } z ^ { - n - 1 } }
\end{array} , \text { where } \left\{\begin{array}{l}
B_{n}=\sum_{\sigma} \alpha_{n}^{\sigma} \\
\beta_{n}=\sum_{\sigma} \sigma \alpha_{n}^{\sigma}
\end{array} .\right.\right.
$$

In terms of $s u(2)$ current algebra, given the two fermionic fields $\psi_{1}=\psi_{\uparrow}$ and $\psi_{2}=\psi_{\downarrow}$, the spinor field $\Psi=\left(\psi_{1} \psi_{2}\right)^{T}$ and the currents $J^{k}=\Psi^{\dagger} \frac{\sigma^{k}}{2} \Psi$, the total theory has an energy momentum tensor with central charge $c=-4$. Its decomposition into a level $k=1 s u(2)$ current algebra $\left\{J_{m}^{k}\right\}$ and a Heisenberg algebra $\left\{B_{n}\right\}$ is such that $T_{m}$ is composed of two Virasoro algebras, one related to the currents $J$, with charge $c=1$, and one related to "free" bosons with charge $c=-5$

$$
T_{m}=L_{m}^{J}+L_{m}^{B}
$$

where

$$
\begin{aligned}
L_{m}^{B} & =\frac{1}{4} \sum_{n \in \mathbb{Z}}: B_{-n} B_{n+m}:+\frac{1}{2}(m+1) B_{m} \\
L_{m}^{J} & =\frac{1}{4} \sum_{e \in \mathbb{Z}}: \beta_{-m} \beta_{n+m}: .
\end{aligned}
$$

We can now expand the bosonized hamiltonian in terms of modes. We go back to the expression for the bosonized hamiltonian (20), and, by separating the several terms and by 
making use of the expansion (28), the first term becomes:

$$
H_{1} \equiv(1-\beta)\left\{\left(B_{0}+1\right)\left(\frac{1}{3} L_{0}^{B}+L_{0}^{J}\right)+\sum_{n \geq 1}\left(B_{-n}\left(\frac{1}{3} L_{n}^{B}+L_{n}^{J}\right)+\left(\frac{1}{3} L_{-n}^{B}+L_{-n}^{J}\right) B_{n}\right)\right\} .
$$

For the second term we have:

$$
H_{2} \equiv \frac{\beta}{2}\left\{2 \sum_{n \geq 1} n B_{-n} B_{n}-2 \sum_{k \geq 1}\left(L_{-k}^{B} B_{k}-B_{-k} L_{k}^{B}+k B_{-k} B_{k}\right)-2 \sum_{k \geq 1}\left(L_{-k}^{J} B_{k}-B_{-k} L_{k}^{J}\right)\right\} .
$$

Finally, the third term will become

$$
H_{3} \equiv-\frac{3 \beta}{2} \sum_{n \geq 1} n B_{-n} B_{n}-2 \beta \sum_{k=1}^{3} \sum_{n \geq 1} n J_{-n}^{k} J_{n}^{k}
$$

The final result for the total hamiltonian in terms of boson (and current) modes is:

$$
\begin{aligned}
\tilde{H}=(\beta-1) & \left(B_{0}+1\right)\left(\frac{1}{3} L_{0}^{B}+L_{0}^{J}\right)+\frac{1+2 \beta}{3} \sum_{n \geq 1} B_{-n} L_{n}^{B}+\frac{1-4 \beta}{3} \sum_{n \geq 1} L_{-n}^{B} B_{n}+ \\
& +\sum_{n \geq 1} B_{-n} L_{n}^{J}+(1-2 \beta) \sum_{n \geq 1} L_{-n}^{J} B_{n}-\frac{3 \beta}{2} \sum_{n \geq 1} n B_{-n} B_{n}-2 \beta \sum_{n \geq 1} n J_{-n} \cdot J_{n} .
\end{aligned}
$$

We now comment on the relation of the above bosonized Hamiltonian to the various bosonized versions of the model studied in the literature. First, by a simple change of our notation :

$$
\beta \rightarrow-\frac{1}{\alpha} \quad ; \quad H_{U}=\alpha \tilde{H}
$$

we obtain a form analogous to one constructed by Uglov in [10]

$$
\begin{aligned}
H_{U}= & E_{0}+(\alpha+1)\left(B_{0}+1\right)\left(\frac{1}{3} L_{0}^{B}+L_{0}^{J}\right)+\frac{\alpha-2}{3} \sum_{n \geq 1} B_{-n} L_{n}^{B}+\frac{\alpha+4}{3} \sum_{n \geq 1} L_{-n}^{B} B_{n} \\
& +\alpha \sum_{n \geq 1} B_{-n} L_{n}^{J}+(\alpha+2) \sum_{n \geq 1} L_{-n}^{J} B_{n}+\frac{3}{2} \sum_{n \geq 1} n B_{-n} B_{n}+2 \sum_{k} \sum_{n \geq 1} n J_{-n}^{k} J_{n}^{k},
\end{aligned}
$$

with

$$
\left[B_{n}, B_{m}\right]=2 n \delta_{n+m, 0}
$$

One can also see that the bosonized hamiltonian described above fits into a (more symmetric) 
form of the representation originally constructed in [12] which reads:

$$
H_{A J L}=\frac{b}{6 q} \sum_{n_{i}} \alpha_{n_{1}} \alpha_{n_{2}} \alpha_{n_{3}} \delta_{n_{1}+n_{2}+n_{3}, 0}+b \sum_{n} \alpha_{n} L_{-n}^{J}+\frac{1}{q} \sum_{n>0} n \alpha_{-n} \alpha_{n}+\sum_{n>0} n J_{-n}^{k} J_{n}^{k}
$$

This is the most general form of the hamiltonian, and the modes $\alpha_{n}$ obey the commutation relation:

$$
\left[\alpha_{n}, \alpha_{m}\right]=q n \delta_{m+n, 0},
$$

In order to exhibit the precise comparison, we will collect terms which have the same number of creation-annihilation operators: starting from (33), we denote the cubic term of $H_{U}$ by $H_{U}^{3}$, the quadratic term in $B_{n}$ by $H_{U}^{2}$, the terms linear in $B_{n}$ and $L_{n}^{J}$ by $H_{U}^{1, J}$, and finally the pure current term will be denoted by $H_{U}^{J}$.

The following change in the normalization of the fields and their commutation relations will be needed perform the comparison:

$$
\begin{aligned}
B_{n}^{\prime} & =(\alpha+2) B_{n}, \quad n \geq 1 \\
B_{-n}^{\prime} & =\alpha B_{-n} \\
{\left[B_{n}^{\prime}, B_{m}^{\prime}\right] } & =2 \alpha(\alpha+2) n \delta_{n+m, 0} .
\end{aligned}
$$

From now on, we are going to drop the primes and will use the same notation for the new fields. In terms of the new fields, $H_{U}$ is given by the sum of the following terms:

$$
\begin{aligned}
H_{U}^{3} & =\frac{1}{4 \alpha(\alpha+2)} \sum_{n \geq 1}\left(B_{-n} B_{-m} B_{n+m}+B_{-n-m} B_{n} B_{m}\right) \\
H_{U}^{2} & =\frac{1}{2 \alpha(\alpha+2)} \sum_{n \geq 1} n B_{-n} B_{n}+\frac{\alpha+1}{2 \alpha(\alpha+2)}\left(B_{0}+1\right) \sum_{n \geq 1} B_{-n} B_{n}, \\
H_{U}^{1, J} & =\sum_{n \geq 1}\left(B_{-n} L_{n}^{J}+L_{-n}^{J} B_{n}\right)+(\alpha+1)\left(B_{0}+1\right) L_{0}^{J}, \\
H_{U}^{J} & =2 \sum_{n \geq 1} \sum_{k} n J_{-n}^{k} J_{n}^{k} .
\end{aligned}
$$

Now, let us consider the hamiltonian (35). We will use a similar notation to refer to the cubic, quadratic, linear and pure current terms in the hamiltonian. Changing the normalization of the $\alpha_{n}$

$$
\begin{aligned}
\alpha_{n}^{\prime} & =b \alpha_{n}, \quad \forall n \\
{\left[\alpha_{n}^{\prime}, \alpha_{m}^{\prime}\right] } & =b^{2} q n \delta_{n+m, 0}
\end{aligned}
$$


and again dropping the primes on the new fields, $H_{A J L}$ will then be the sum of:

$$
\begin{aligned}
H_{A J L}^{3} & =\frac{1}{2 b^{2} q} \sum_{n, m \geq 1}\left(\alpha_{-n} \alpha_{-m} \alpha_{n+m}+\alpha_{-n-m} \alpha_{n} \alpha_{m}\right) \\
H_{A J L}^{2} & =\frac{1}{b^{2} q} \sum_{n \geq 1} n \alpha_{-n} \alpha_{n}+\frac{1}{b^{2} q} \alpha_{0} \sum_{n \geq 1} \alpha_{-n} \alpha_{n}, \\
H_{A J L}^{1, J} & =\sum_{n \geq 1}\left(\alpha_{-n} L_{n}^{J}+L_{-n}^{J} \alpha_{n}\right)+\alpha_{0} L_{0}^{J}, \\
H_{A J L}^{J} & =\sum_{n \geq 1} \sum_{k} n J_{-n}^{k} J_{n}^{k} .
\end{aligned}
$$

The relation between $B_{n}$ and $\alpha_{n}$ can be determined. In fact, if the following relation holds, the two hamiltonians become equivalent with the same commutation relations.

$$
\begin{aligned}
b^{2} q & =2 \alpha(\alpha+2) \\
\alpha_{0} & =(\alpha+1)\left(B_{0}+1\right) .
\end{aligned}
$$

The factor of 2 in $H_{U}^{J}$ is not present in $H_{A J L}^{J}$. That is due to a different definition of the current modes. We can see that there is perfect agreement between the two hamiltonians. With this we have also demonstrated that the bosonized hamiltonian $H_{U}$, is hermitian.

One last comment should be made. If we write the hamiltonian (31) in terms of the original bosonization fields $\alpha^{\sigma} \equiv \phi^{\sigma}$ we will be able to make contact with the collective field approach which will be summarized in section 4, As a final comment on the current algebra representation we mention the following. Here we have described in detail the formalism in the simplest $\mathrm{SU}(2)$ case for purposes of comparing different approaches and describing some nontrivial applications. As we have mentioned the formalism for general SU(R) was developed in [12] . Of considerable interest is the case $R=N$ with $N \rightarrow \infty$. The current algebraic representation is well suited for that since it leads to a large $N$ limit of WZW models, a well studied subject. Through the large $N$ limit of the current algebra one realizes a $W_{\infty}$ algebra, the expected symmetry of the matrix model [45]. Aspects of the $R=N \rightarrow \infty$ theory were already given in [44] .

\section{Collective Field Theory}

For obtaining the collective field theory representation one considers a permutation symmetric version of the model with the idea of expressing the theory in terms of collective densities. 
Considering the original hamiltonian (12), given by:

$$
H=\sum_{i} D_{i}^{2}-\sum_{i \neq j} \frac{z_{i} z_{j}}{\left(z_{i}-z_{j}\right)^{2}} \beta\left(\beta+P_{i j}\right)
$$

we therefore need to perform a similarity transformation to bring the theory into a bosonic picture. We write the wavefunctions of $H$ as $\Psi=\Psi_{0} \chi$, with $\chi$ being a bosonic function. The form of $\Psi_{0}$ defines then the statistics of the model. In the previous construction we performed a transformation to the fermionic picture by using $\Psi_{0}=\Delta^{\beta}$. To obtain a bosonic picture, we will now require an extra determinant factor in the similarity transformation, $\Psi_{0}=\Delta^{\beta+1}$. In that case, the gauged hamiltonian is:

$$
H_{\beta+1}=\underbrace{\sum_{i} D_{i}^{2}}_{H_{\beta+1}^{1}}+\underbrace{\frac{(\beta+1)}{2} \sum_{i \neq j} \frac{z_{i}+z_{j}}{z_{i}-z_{j}}\left(D_{i}-D_{j}\right)}_{H_{\beta+1}^{2}} \underbrace{-2 \beta \sum_{i<j} \theta_{i j} \theta_{j i}\left[1-P_{i j}\right]}_{H_{\beta+1}^{s}}+E_{G S}(\beta+1),
$$

where $\theta_{i j} \equiv \frac{z_{i}}{z_{i}-z_{j}}$. The ground state energy is given by $E_{G S}(\beta+1)=(\beta+1)^{2} \frac{N\left(N^{2}-1\right)}{12}$.

In the last section we bosonized the hamiltonian $\tilde{H}$ in terms of current fields [11, 12]. In this section we describe in some detail the collective field theory approach. This method was useful for various matrix model problems and in particular systems of Calogero-type [24, 49, 50]. In the case of a spin-Calogero model one can similarly obtain the hamiltonian in terms of collective modes in momentum space [13]. ${ }^{4}$ We will also want to obtain the same result in terms of collective fields in coordinate space, as it will be useful in further perturbative and nonperturbative studies.

We will start from the hamiltonian $H_{\beta+1}$ (one could get a similar result by starting from $\left.\tilde{H} \equiv H_{\beta}\right)$. One defines collective densities

$$
\phi_{\sigma}(x)=\sum_{i=1}^{N} \delta\left(x-x_{i}\right) \delta_{\sigma \sigma_{i}} \quad, \quad \phi(x)=\sum_{\sigma} \phi_{\sigma}(x)
$$

and its conjugate momentum $\Pi^{\sigma}(x)=\frac{\delta}{\delta \phi_{\sigma}(x)} .{ }^{5}$ We take $\Pi^{\sigma}(x)$ to be non-hermitian just to save writing $i$ s. In terms of Fourier modes, the fields defined above become (with $n$ integer because we are on the circle):

$$
\phi_{n}^{\sigma}=\int_{-\pi}^{\pi} d x e^{i n x} \phi_{\sigma}(x)=\sum_{i=1}^{N} z_{i}^{n} \delta_{\sigma \sigma_{i}} \quad, \quad \Pi_{n}^{\sigma}=\frac{\partial}{\partial \phi_{n}^{\sigma}}=\int \frac{d x}{2 \pi} e^{-i n x} \frac{\partial}{\partial \phi_{\sigma}(x)},
$$

\footnotetext{
${ }^{4}$ Note that the gauged hamiltonian found in 13 is related to (45) by changing, in the latter, $\beta+1$ to $\beta$ on the second term. The discrepancy comes from a difference on the starting point of each case.

${ }^{5}$ In this section coordinate $x$ has range on the interval $[-\pi, \pi]$ unless said otherwise. Also, the spin variable $\sigma$ has possible values $\sigma= \pm 1$.
} 
The expressions that follow are obtained through a change of variables following the standard collective field techniques [51]. We start from (13) and define $\hat{\Pi}_{n}^{\sigma} \equiv n \frac{\partial}{\partial \phi_{n}^{\sigma}}=n \Pi_{n}^{\sigma}$ (such that $\left.\left[\phi_{n}^{\sigma}, \hat{\Pi}_{m}^{\sigma^{\prime}}\right]=-m \delta_{\sigma \sigma^{\prime}} \delta_{m, n}\right)$. Writing the dependence on spin explicitly, the derivative $D_{i}$ is given by chain rule:

$$
D_{i}=z_{i} \frac{\partial}{\partial z_{i}}=\sum_{\sigma} \sum_{n} z_{i} \frac{\partial \phi_{n}^{\sigma}}{\partial z_{i}} \frac{\partial}{\partial \phi_{n}^{\sigma}}=\sum_{\sigma} \sum_{n} z_{i} \frac{\partial}{\partial z_{i}}\left(\sum_{j} z_{j}^{n} \delta_{\sigma \sigma_{j}}\right) \frac{\partial}{\partial \phi_{n}^{\sigma}}=\sum_{\sigma} \sum_{n} z_{i}^{n} \delta_{\sigma \sigma_{i}} \hat{\Pi}_{n}^{\sigma} .
$$

This implies that for $H_{1}$ we have

$$
\begin{aligned}
H_{1} & =\sum_{i} D_{i}^{2}=\sum_{\sigma} \sum_{i} \sum_{n} \delta_{\sigma \sigma_{i}} z_{i}\left\{\frac{\partial z_{i}^{n}}{\partial z_{i}} \hat{\Pi}_{n}^{\sigma}+z_{i}^{n} \sum_{\sigma^{\prime}} \sum_{m} \frac{\partial \phi_{m}^{\sigma^{\prime}}}{\partial z_{i}} \frac{\partial}{\partial \phi_{m}^{\sigma^{\prime}}} \hat{\Pi}_{n}^{\sigma}\right\} \\
& =\sum_{\sigma}\left\{\sum_{n} n \phi_{n}^{\sigma} \hat{\Pi}_{n}^{\sigma}+\sum_{m, n} \phi_{n+m}^{\sigma} \hat{\Pi}_{m}^{\sigma} \hat{\Pi}_{n}^{\sigma}\right\} .
\end{aligned}
$$

Similarly for $H_{2}$

$$
\begin{aligned}
H_{2} & =\frac{\beta+1}{2} \sum_{i \neq j} \frac{z_{i}+z_{j}}{z_{i}-z_{j}}\left(D_{i}-D_{j}\right) \\
& =(\beta+1)\left(\sum_{\sigma=\sigma^{\prime}}+\sum_{\sigma \neq \sigma^{\prime}}\right) \sum_{i \neq, j} \frac{z_{i}}{z_{i}-z_{j}} \sum_{m}\left(z_{i}^{m} \delta_{\sigma \sigma_{i}} \delta_{\sigma^{\prime} \sigma_{j}}-z_{j}^{m} \delta_{\sigma^{\prime} \sigma_{i}} \delta_{\sigma \sigma_{j}}\right) \hat{\Pi}_{m}^{\sigma} .
\end{aligned}
$$

The term with $\sigma=\sigma^{\prime}$ is:

$$
H_{\sigma}^{2}=(\beta+1) \sum_{\sigma}\left\{\sum_{n, m \geq 1} \phi_{n}^{\sigma} \phi_{m}^{\sigma} \hat{\Pi}_{m+n}^{\sigma}+\sum_{m \geq 1}\left(\phi_{0}^{\sigma}-m\right) \phi_{m}^{\sigma} \hat{\Pi}_{m}^{\sigma}\right\}
$$

But the term with $\sigma \neq \sigma^{\prime}$ is more complicated. Doing some algebra leads to (notice that $\phi_{n}$ is not defined for $n$ negative):

$$
H_{\sigma \neq \sigma^{\prime}}^{2}=(\beta+1) \sum_{\sigma \neq \sigma^{\prime}}\left(\sum_{m, n \geq 1} \phi_{n}^{\sigma^{\prime}} \phi_{m}^{\sigma} \hat{\Pi}_{m+n}^{\sigma}+\sum_{m>0} \phi_{0}^{\sigma} \phi_{m}^{\sigma^{\prime}} \hat{\Pi}_{m}^{\sigma}\right)
$$

A related result can be found in [13]. In fact the terms written above agree with the corresponding ones from 13 .

One is also interested in the expressions for the hamiltonian in coordinate space, as these will play an important role when performing small fluctuations around a classical background. This will be explored further in the sections below. For now let us express the hamiltonian in terms 
of density fields. For the $H_{1}$ we get

$$
H_{1}=-\sum_{i} \frac{\partial^{2}}{\partial x_{i}^{2}}=-\sum_{\sigma} \int d x \phi_{\sigma}(x) \partial^{2} \Pi^{\sigma}(x)-\sum_{\sigma} \int d x \phi_{\sigma}(x) \partial \Pi^{\sigma}(x) \partial \Pi^{\sigma}(x) .
$$

To determine $H_{2}$ we need to write it as

$$
H_{2}=\underbrace{\frac{\beta+1}{2} \sum_{i, j}\left(\theta_{i j}-\theta_{j i}\right)\left(D_{i}-D_{j}\right)}_{H_{21}} \underbrace{-\frac{\beta+1}{2} \sum_{i=j} \lim _{z_{i} \rightarrow z_{j}} \frac{z_{i}+z_{j}}{z_{i}-z_{j}}\left(D_{i}-D_{j}\right)}_{H_{22}},
$$

and each term can be found to be:

$$
\begin{aligned}
& H_{21}=-\frac{\beta+1}{2} \sum_{\sigma, \sigma^{\prime}} \int d x \phi_{\sigma}(x)\left(\int d y \phi_{\sigma^{\prime}}(y) \cot \left(\frac{x-y}{2}\right)\right)\left[\partial \Pi^{\sigma}(x)-\partial \Pi^{\sigma^{\prime}}(y)\right], \\
& H_{22}=(\beta+1) \int d x\left(\partial^{2} \phi_{\sigma}\right)(x) \Pi^{\sigma}(x)=(\beta+1) \int d x \phi_{\sigma}(x) \partial^{2} \Pi^{\sigma}(x) .
\end{aligned}
$$

The term $H_{s}$ can also be expressed in terms of collective fields. The operator $P_{i j}^{\sigma \sigma^{\prime}}$ can be defined by how it acts on $\phi_{n}^{\sigma}$. We know that the effective hamiltonian acts on wavefunctions that depend solely on $\phi_{n}^{\sigma}$ (bosonic functions), so we want to determine the action of $P_{i j}$ on $\chi\left(\phi_{n}^{\sigma}\right)$. First,

$$
P_{i j} \phi_{n}^{\sigma}=P_{i j} \sum_{\ell=1}^{N} z_{\ell}^{n} \delta_{\sigma \sigma_{\ell}}=\phi_{n}^{\sigma}+z_{i}^{n}\left(\delta_{\sigma, \sigma_{j}}-\delta_{\sigma, \sigma_{i}}\right)+z_{j}^{n}\left(\delta_{\sigma, \sigma_{i}}-\delta_{\sigma, \sigma_{j}}\right) .
$$

So $P_{i j}$ acts as a translation operator, and when acting on a general bosonic wavefunction $\chi\left(\phi_{n}^{\sigma}\right)$, we have

$$
P_{i j} \chi\left(\phi_{n}^{\sigma}\right)=e^{\left\{P\left(x_{i}, \sigma_{i} ; x_{j}, \sigma_{j}\right)\right\}} \chi\left(\phi_{n}^{\sigma}\right) .
$$

where

$$
\begin{aligned}
P\left(x_{i}, \sigma_{i} ; x_{j}, \sigma_{j}\right) & \equiv \sum_{\sigma}\left(\delta_{\sigma \sigma_{i}}-\delta_{\sigma \sigma_{j}}\right)\left(\Pi^{\sigma}\left(x_{i}\right)-\Pi^{\sigma}\left(x_{j}\right)\right) \\
& =\int_{x_{j}}^{x_{i}} d x\left(\partial_{x} \Pi^{\sigma_{i}}(x)-\partial_{x} \Pi^{\sigma_{j}}(x)\right) .
\end{aligned}
$$


Finally, $H_{s}$ can be rewritten as ${ }^{6}$

$$
\begin{aligned}
H_{s} & =-\beta \sum_{i \neq j} \frac{z_{i} z_{j}}{\left(z_{i}-z_{j}\right)^{2}}\left(P_{i j}-1\right) \\
& =\frac{\beta}{4} \sum_{\sigma, \sigma^{\prime}} \int d x \int d y \phi_{\sigma}(x) \sin ^{-2}\left(\frac{x-y}{2}\right)\left\{\phi_{\sigma^{\prime}}(y)-\delta_{\sigma \sigma^{\prime}} \delta(y-x)\right\}\left\{e^{P\left(x, \sigma ; y, \sigma^{\prime}\right)}-1\right\} \\
& =\frac{\beta}{4} \sum_{\sigma, \sigma^{\prime}} \int d x \int d y \phi_{\sigma}(x) \sin ^{-2}\left(\frac{x-y}{2}\right) \phi_{\sigma^{\prime}}(y)\left\{\sum_{n=1}^{\infty} \frac{1}{n !}\left[\int_{y}^{x} d z \partial_{z}\left(\Pi^{\sigma}(z)-\Pi^{\sigma^{\prime}}(z)\right)\right]^{n}\right\} .
\end{aligned}
$$

Note that this interaction term vanishes for $\sigma=\sigma^{\prime}$, so there is no issue at $y=x$. In fact the sum over both $\sigma, \sigma^{\prime}$ could be substituted by a sum over $\sigma \neq \sigma^{\prime}$.

To complete the comparison with [13], we still need to confirm this last term of the hamiltonian, $H_{s}$.

As it was seen in (49), the operator $P_{i j}$ acts on bosonic wave functions as:

$$
e^{P\left(x, \sigma ; y, \sigma^{\prime}\right)}=\exp \left\{\sum_{n} \frac{1}{n}\left(z_{x}^{n}-z_{y}^{n}\right)\left(\hat{\Pi}_{n}^{\sigma}-\hat{\Pi}_{n}^{\sigma^{\prime}}\right)\right\}=:\left(P_{x}^{\sigma \sigma^{\prime}}\right)\left(P_{y}^{\sigma \sigma^{\prime}}\right)^{-1}:
$$

where

$$
P_{x}^{\sigma \sigma^{\prime}}=\exp \left\{\sum_{n} \frac{1}{n} z_{x}^{n}\left(\hat{\Pi}_{n}^{\sigma}-\hat{\Pi}_{n}^{\sigma^{\prime}}\right)\right\}=\exp \left\{\Pi^{\sigma}\left(z_{x}\right)-\Pi^{\sigma^{\prime}}\left(z_{x}\right)\right\},
$$

and $z_{x} \equiv e^{i x}$. If we go to complex coordinates, ${ }^{7}$ we get (as before, we have to be careful in the

${ }^{6}$ In order to write this and other sums that diverge when $i=j$, we use the following result. Let $f\left(x_{i}, x_{j}\right)$ be a function that describes some 2-body interaction, either singular or not when $i \rightarrow j$. Then

$$
\begin{aligned}
\sum_{i \neq j} f\left(x_{i}, \sigma_{i} ; x_{j}, \sigma_{j}\right) & =\sum_{i \neq j} \sum_{\sigma, \sigma^{\prime}} \int d x \delta_{\sigma \sigma_{i}} \delta_{\sigma^{\prime} \sigma_{j}} \delta\left(x-x_{i}\right) \int d y \delta\left(y-x_{j}\right) f\left(x, \sigma ; y, \sigma^{\prime}\right) \\
& =\sum_{\sigma, \sigma^{\prime}} \int d x \int d y f\left(x, \sigma ; y, \sigma^{\prime}\right)\left\{\sum_{i, j}-\sum_{i=j}\right\} \delta_{\sigma \sigma_{i}} \delta_{\sigma^{\prime} \sigma_{j}} \delta\left(x-x_{i}\right) \delta\left(y-x_{j}\right) \\
& =\sum_{\sigma, \sigma^{\prime}} \int d x \int d y \phi_{\sigma}(x) f\left(x, \sigma ; y, \sigma^{\prime}\right)\left\{\phi_{\sigma^{\prime}}(y)-\delta_{\sigma \sigma^{\prime}} \delta(y-x)\right\} .
\end{aligned}
$$

This result allows us to regularize the integrals, once we use the density formulation. One other way of writing this result is by the use of principal value integrals:

$$
\sum_{i \neq j} f\left(x_{i}, \sigma_{i} ; x_{j}, \sigma_{j}\right)=\sum_{\sigma, \sigma^{\prime}} \int d x f d y \phi_{\sigma}(x) f\left(x, \sigma ; y, \sigma^{\prime}\right) \phi_{\sigma^{\prime}}(y) .
$$

${ }^{7}$ In order to go to complex coordinates, remember that $\phi_{\sigma}(x)$ has conformal weight one, much like $\psi^{\dagger}$ in the previous section, and so transforms like $\phi_{\sigma}(x) \rightarrow z \phi_{\sigma}(z)$. 
integration, as the integration over $w$ will be around the coordinate $z$ )

$$
\begin{aligned}
H_{s} & =\beta \sum_{\sigma \neq \sigma^{\prime}} \oint d z \oint d w \frac{z w}{(z-w)^{2}} \phi_{\sigma}(z) \phi_{\sigma^{\prime}}(w)\left\{e^{P\left(z, \sigma ; w, \sigma^{\prime}\right)}-1\right\} \\
& =\beta \sum_{\sigma \neq \sigma^{\prime}} \oint d z \oint d w \frac{1}{(z-w)^{2}}: z \phi_{\sigma}(z) e^{\Pi^{\sigma}(z)-\Pi^{\sigma^{\prime}}(z)} w \phi_{\sigma^{\prime}}(w) e^{-\Pi^{\sigma}(z)+\Pi^{\sigma^{\prime}}(z)}: .
\end{aligned}
$$

Some simplifications in the above expression were due to restricting the expansion of $\phi$ to positive modes: $\phi_{\sigma}(z)=\sum_{n \geq 0} \phi_{n}^{\sigma} z^{-n-1}$. There is one other way of writing this result:

$$
\begin{aligned}
H_{s} & =\beta \sum_{\sigma \neq \sigma^{\prime}} \oint \frac{d \eta}{\eta} \oint \frac{d \xi}{\xi} \sum_{k, \ell \geq 0} \eta^{k} \xi^{\ell} \int d x \phi_{\sigma}(x) z_{x}^{k} \int d y \phi_{\sigma^{\prime}}(y) z_{y}^{\ell} \frac{z_{x} z_{y} e^{\sum_{n>0} \frac{1}{n}\left(\eta^{-n}-\xi^{-n}\right)\left(\hat{\Pi}_{n}^{\sigma}-\hat{\Pi}_{n}^{\sigma^{\prime}}\right)}}{\left(z_{x}-z_{y}\right)^{2}} \\
& =\frac{\beta}{2} \sum_{\sigma \neq \sigma^{\prime}} \oint \frac{d \eta}{\eta} \oint \frac{d \xi}{\xi} \sum_{k, \ell \geq 0} \eta^{k} \xi^{\ell} \phi_{k}^{\sigma} \phi_{\ell}^{\sigma^{\prime}} e^{\sum_{n>0} \frac{1}{n}\left(\eta^{-n}-\xi^{-n}\right)\left(\hat{\Pi}_{n}^{\sigma}-\hat{\Pi}_{n}^{\sigma^{\prime}}\right)} \sum_{m>0} m\left[\left(\frac{\eta}{\xi}\right)^{m}+\left(\frac{\xi}{\eta}\right)^{m}\right] .
\end{aligned}
$$

These results represent the collective field bosonization of the C-S many body problem. They are in accordance with the expressions found in [13]. One can further ask regarding a connection with the bosonization procedure of section 3 . In one case we dealt with a fermionic effective hamiltonian and followed standard rules of bosonization. In the other one first went to a bosonic picture and then applied the technique of collective fields. Since the relation between the fermionic and the bosonic pictures can be described in terms of a single similarity transformation it is expected that the two versions of the continuum hamiltonian are equivalent. We can expect that the identification can be demonstrated by a field theoretic similarity transformation. Its explicit form remains to be understood.

To conclude this section, we now present the total collective hamiltonian corresponding to the spin Calogero-Sutherland model, in coordinate space:

$$
\begin{aligned}
H=E+\beta \sum_{\sigma} & \int d x \phi_{\sigma}(x) \partial^{2} \Pi^{\sigma}(x)-\sum_{\sigma} \int d x \phi_{\sigma}(x) \partial \Pi^{\sigma}(x) \partial \Pi^{\sigma}(x)- \\
& -(\beta+1) \sum_{\sigma, \sigma^{\prime}} \int d x \phi_{\sigma}(x) \int d y \phi_{\sigma^{\prime}}(y) \cot \left(\frac{x-y}{2}\right) \partial \Pi^{\sigma}(x)+ \\
+ & \frac{\beta}{4} \sum_{\sigma, \sigma^{\prime}} \int d x \int d y \frac{\phi_{\sigma}(x) \phi_{\sigma^{\prime}}(y)}{\sin ^{2}\left(\frac{x-y}{2}\right)}\left\{\sum_{n=1}^{\infty} \frac{1}{n !}\left[\int_{y}^{x} d z \partial_{z}\left(\Pi^{\sigma}(z)-\Pi^{\sigma^{\prime}}(z)\right)\right]^{n}\right\} .
\end{aligned}
$$

The hamiltonian thus presented is comparable to what was obtained by the bosonization procedure of section 3, given by (20), and will be used in the following sections. 


\section{Spectrum Equation}

The next two sections will be devoted to the study of some applications of the bosonized spin CS hamiltonian. In this section, we will take a second look at the collective form of the hamiltonian, and put it in terms of spin and charge bosons, as was done in section 3 of the paper. In this form of the hamiltonian, we can use semi-classical method of determining the energies, in order to be able to perform small fluctuations. This will result in an eigenvalue problem of the Marchesini-Onofri type. Then, in section [6] we will describe the formalism in an example given by a nontrivial background based on exact eigenstates of [21].

\subsection{The Marchesini-Onofri kernel}

We now go back to the effective hamiltonian $H_{\beta+1}$, in its collective form (52), but will regularize the integrals by the use of principal value integrals. It is easy to check that, this be the case, (52) can be rewritten as:

$$
\begin{aligned}
H=E-\sum_{\sigma} & \int d x \phi_{\sigma}(x) \partial^{2} \Pi^{\sigma}(x)-\sum_{\sigma} \int d x \phi_{\sigma}(x) \partial \Pi^{\sigma}(x) \partial \Pi^{\sigma}(x)- \\
& -(\beta+1) \sum_{\sigma, \sigma^{\prime}} \int d x \phi_{\sigma}(x) f d y \phi_{\sigma^{\prime}}(y) \cot \left(\frac{x-y}{2}\right) \partial \Pi^{\sigma}(x)+ \\
& \frac{\beta}{4} \sum_{\sigma \neq \sigma^{\prime}} \int d x f d y \phi_{\sigma}(x) \sin ^{-2}\left(\frac{x-y}{2}\right) \phi_{\sigma^{\prime}}(y)\left\{e^{P\left(x^{\sigma}, y^{\sigma^{\prime}}\right)}-1\right\},
\end{aligned}
$$

with $E=(\beta+1)^{2} \frac{N\left(N^{2}-1\right)}{12}$ and

$$
P\left(x, \sigma ; y, \sigma^{\prime}\right)=\int_{y}^{x} d z \partial_{z}\left(\Pi^{\sigma}(z)-\Pi^{\sigma^{\prime}}(z)\right)=\left(\Pi^{\sigma}-\Pi^{\sigma^{\prime}}\right)(x)-\left(\Pi^{\sigma}-\Pi^{\sigma^{\prime}}\right)(y) .
$$

The next step is to introduce the spin and charge bosons, just like we did in section 3 . Remembering that we are dealing with an $s u(2)$ current algebra, let

$$
\phi=\sum_{\sigma} \phi_{\sigma} \quad ; \quad \psi=\sum_{\sigma} \sigma \phi_{\sigma}
$$

Then, as a consequence of $\Pi_{\sigma} \equiv \frac{\partial}{\partial \phi_{\sigma}}$, we have

$$
\Pi_{\phi} \equiv \frac{\delta}{\delta \phi}=\sum_{\sigma} \Pi_{\sigma} \quad ; \quad \Pi_{\psi} \equiv \frac{\delta}{\delta \psi}=\sum_{\sigma} \sigma \Pi_{\sigma}
$$

With this definitions we can rewrite the hamiltonian in terms of the new fields (by using 
that $\phi_{\sigma}=\frac{1}{2}(\phi+\sigma \psi)$ and $\left.\Pi_{\sigma}=\frac{1}{2}\left(\Pi_{\phi}+\sigma \Pi_{\psi}\right)\right)$. Recalling equations (47), (48) and (51) one can easily obtain the following results:

$$
\begin{aligned}
H_{1}= & -\frac{1}{2} \int d x\left(\partial^{2} \phi \Pi_{\phi}+\partial^{2} \psi \Pi_{\psi}\right)-\frac{1}{2} \int d x f d y \delta(x-y) \psi(x) \partial \Pi_{\phi}(x) \partial \Pi_{\psi}(y) \\
& -\frac{1}{4} \int d x f d y \delta(x-y) \phi(x)\left[\partial \Pi_{\phi}(x) \partial \Pi_{\phi}(y)+\partial \Pi_{\psi}(x) \partial \Pi_{\psi}(y)\right], \\
H_{2}= & \frac{\beta+1}{2} \int d x\left(\partial^{2} \phi \Pi_{\phi}+\partial^{2} \psi \Pi_{\psi}\right) \\
& -\frac{\beta+1}{2} \int d x f d y \phi(y) \cot \left(\frac{x-y}{2}\right)\left[\phi(x) \partial \Pi_{\phi}(x)+\psi(x) \partial \Pi_{\psi}(x)\right] .
\end{aligned}
$$

The only term left to determine is $H_{s}$. Because we only have two spins $(\sigma= \pm 1)$, the sum over $\sigma \neq \sigma^{\prime}$ in $H_{s}$ can be substituted by a sum $\sigma>\sigma^{\prime}$ (which is not really a sum, as the only possibility is $\sigma=1, \sigma^{\prime}=-1$ ). Then using

$$
P(x, \uparrow ; y, \downarrow)=\left(\Pi^{\uparrow}-\Pi^{\downarrow}\right)(x)-\left(\Pi^{\uparrow}-\Pi^{\downarrow}\right)(y)=\Pi_{\psi}(x)-\Pi_{\psi}(y),
$$

we can write $H_{s}$ in the following way:

$$
H_{s}=\frac{\beta}{2} \sum_{\sigma>\sigma^{\prime}} \int d x f d y \phi_{\uparrow}(x) \phi_{\downarrow}(y) \sin ^{-2}\left(\frac{x-y}{2}\right)\left\{e^{\Pi_{\psi}(x)-\Pi_{\psi}(y)}-1\right\}=H_{s}^{1}+H_{s}^{2},
$$

where after some algebra one gets:

$$
\begin{aligned}
& H_{s}^{1}=-\frac{\beta}{8} \int d x f d y \frac{[\phi(x) \phi(y)-\psi(x) \psi(y)]}{\sin ^{2}\left(\frac{x-y}{2}\right)}\left\{1-e^{\Pi_{\psi}(x)-\Pi_{\psi}(y)}\right\}, \\
& H_{s}^{2}=-\frac{\beta}{4} \int d x f d y \frac{\phi(x) \psi(y)}{\sin ^{2}\left(\frac{x-y}{2}\right)} \sinh \left[\Pi_{\psi}(x)-\Pi_{\psi}(y)\right] .
\end{aligned}
$$

The first conclusion that one can draw is that the interaction term $H_{s}$ does not depend on the field $\Pi_{\phi}$. This means that the full hamiltonian will be at most quadratic in this field, and we can proceed to hermitianize the hamiltonian in terms of the fields $\phi, \Pi_{\phi}$, following the standard procedure shown in [51, 52. To do so, let us first re-write the full hamiltonian in a simpler way:

$$
\begin{aligned}
H= & \int d x \omega^{\phi}(x) \Pi_{\phi}+\int d x \int d y \Omega_{x, y}^{\phi} \Pi_{\phi}(x) \Pi_{\phi}(y)+2 \int d x \int d y \widetilde{\Omega}_{x, y} \Pi_{\phi}(x) \Pi_{\psi}(y)+ \\
& +\int d x \omega^{\psi}(x) \Pi_{\psi}+\int d x \int d y \Omega_{x, y}^{\psi} \Pi_{\psi}(x) \Pi_{\psi}(y)+H_{s}+E
\end{aligned}
$$


where the definitions for these terms are given below:

$$
\begin{aligned}
\omega^{\phi}(x) & =-\frac{1}{2} \partial^{2} \phi(x)+\frac{\beta+1}{2} \partial_{x}\left[\phi(x) f d y \phi(y) \cot \left(\frac{x-y}{2}\right)\right] \\
\omega^{\psi}(x) & =-\frac{1}{2} \partial^{2} \psi(x)+\frac{\beta+1}{2} \partial_{x}\left[\psi(x) f d y \phi(y) \cot \left(\frac{x-y}{2}\right)\right] ; \\
\Omega_{x, y}^{\phi} & =-\frac{1}{4} \partial_{x} \partial_{y}[\delta(x-y) \phi(x)] \\
\Omega_{x, y}^{\psi} & =-\frac{1}{4} \partial_{x} \partial_{y}[\delta(x-y) \phi(x)] \\
\widetilde{\Omega}_{x, y} & =-\frac{1}{4} \partial_{x} \partial_{y}[\delta(x-y) \psi(x)] .
\end{aligned}
$$

As we said, we are interested in hemitianizing the hamiltonian with respect to the fields $\phi, \Pi_{\phi}$. We know from [51, 52] that it can be done by a change of variables, equivalent to the similarity transformation

$$
\phi \rightarrow \phi \quad ; \quad \Pi_{\phi} \rightarrow \Pi_{\phi}-\frac{1}{2} \frac{\partial \ln J}{\partial \phi}
$$

where the Jacobian $J$ obeys

$$
\frac{\partial \ln J}{\partial \phi}=\int d y\left(\Omega^{\phi}\right)_{x, y}^{-1} \omega^{\phi}(y)
$$

With this transformation, the first two terms in (55), i.e. the terms that have no $\Pi_{\psi}$ dependence, become $^{1}$

$$
\begin{gathered}
H_{\phi} \rightarrow-\frac{1}{4} \int d x \partial \Pi_{\phi}(x) \phi(x) \partial \Pi_{\phi}(x)+\frac{(\beta+1)}{2} \int d x \phi(x) \partial_{x}\left[f d y \phi(y) \cot \left(\frac{x-y}{2}\right)\right]+ \\
-\frac{N}{12}(\beta+1)^{2}+\frac{\pi^{2}}{3}(\beta+1)^{2} \int d x \phi^{3}(x)+\frac{\beta^{2}}{4} \int d x \frac{(\partial \phi)^{2}}{\phi}
\end{gathered}
$$

where we have used the following identity (which can be proven by Fourier transform):

$$
\frac{1}{4} \int d x \phi(x)\left[f d y \phi(y) \cot \left(\frac{x-y}{2}\right)\right]^{2}=\frac{\pi^{2}}{3} \int d x \phi^{3}(x)-\frac{N^{3}}{12} \text {. }
$$

But these terms are not the only ones that get changed with the transformation. The third term of (55) also gets shifted, as it has a field $\Pi_{\phi}$ :

$$
\begin{aligned}
H_{\phi \psi} \rightarrow & 2 \int d x \int d y \widetilde{\Omega}_{x, y}\left(\Pi_{\phi}(x)-\frac{1}{2} f d z\left(\Omega_{x, z}^{\phi}\right)^{-1} \omega^{\phi}(z)\right) \Pi_{\psi}(y) \\
= & 2 \int d x \int d y \widetilde{\Omega}_{x, y} \Pi_{\phi}(x) \Pi_{\psi}(y)+ \\
& +\frac{1}{2} \int d x \partial_{x}\left\{\psi(x) \frac{(\partial \phi)}{\phi}-(\beta+1) \psi(x) \int d y \phi(y) \cot \left(\frac{x-y}{2}\right)\right\} \Pi_{\psi}(y) .
\end{aligned}
$$

\footnotetext{
${ }^{1}$ It is easy to show that $\partial_{x} \partial_{y}\left(\Omega_{x, y}^{\phi}\right)^{-1}=-\frac{4}{\phi(x)} \delta(x-y)$.
} 
All the other terms in the hamiltonian do not change under the transformation. The total hamiltonian after the transformation becomes:

$$
\tilde{H}=H_{\phi}+2 \int d x \int d y \widetilde{\Omega}_{x, y} \Pi_{\phi}(x) \Pi_{\psi}(y)+\int d x \tilde{\omega}^{\psi}(x) \Pi_{\psi}+\int d x \int d y \Omega_{x, y}^{\psi} \Pi_{\psi}(x) \Pi_{\psi}(y)+H_{s}
$$

where we have defined all the quantities above, except for the new $\tilde{\omega}^{\psi}$, given by

$$
\tilde{\omega}^{\psi}(x)=\frac{1}{2} \partial_{x}\left(\psi \frac{\partial_{x} \phi}{\phi}-\partial_{x} \psi\right) .
$$

The objective of such a transformation is to be able to study fluctuations about a single matrix background, which is a stationary point of the explicitly hermitian effective potential in the fields $\phi$. As we will be interested in small fluctuations around this stationary point, we can still expand the term $H_{s}$ of the hamiltonian up to third order in the fields (ignoring higher order effects). Keeping only linear, quadratic and cubic terms, we have $H_{s}^{1} \approx 0$, and so

$$
H_{s} \approx-\frac{\beta}{4} \int d x f d y \frac{[\phi(x) \psi(y)-\psi(x) \phi(y)]}{\sin ^{2}\left(\frac{x-y}{2}\right)} \Pi_{\psi}(x) .
$$

We still want to make one other assumption. We will assume that the spin field $\psi$ does not acquire an expectation value in the classical equations of motion, and so we will drop the term with double $\Pi_{\psi} \Pi_{\psi}$ in the hamiltonian, $H_{\psi \psi}$. The reason for this is that this term is directly related to the term of current-current interaction (the $J^{2}$ term in section 3). In fact $\psi$ was seen to be the bosonic field used to bosonize the current terms (in section 3, we called it $\beta$ ) and as the current interaction will be zero at the classical stationary point, the term $H_{\psi \psi}$ of the hamiltonian will not be included in the final version of the hamiltonian.

Finally we write the full hamiltonian as the sum of

$$
H=H_{\phi}+\Delta H
$$

with

$$
\begin{aligned}
\Delta H= & -\frac{1}{2} \int d x \partial \Pi_{\phi}(x) \psi(x) \partial \Pi_{\psi}(x)+ \\
& +\frac{1}{2} \int d x \partial_{x}\left(\psi \frac{\partial_{x} \phi}{\phi}-\partial_{x} \psi\right) \Pi_{\psi}-\frac{\beta}{4} \int d x f d y \frac{[\phi(x) \psi(y)-\psi(x) \phi(y)]}{\sin ^{2}\left(\frac{x-y}{2}\right)} \Pi_{\psi}(x) .
\end{aligned}
$$

As mentioned before, our intention is to consider small fluctuations around a classical background field configuration. The term $H_{\phi}$ of the hamiltonian allows us, in the large $N$ limit, to determine the classical background $\phi(x)=\phi_{0}(x)$, with $\psi_{0}=0$ (solving the variation associated with $H_{\phi}$, and ignoring several terms which are higher order in $1 / N[24,53,54]$ ). Also, to obtain 
$\phi_{0}$ one imposes that $\Pi_{\psi}$ doesn't acquire a classical expectation value. The ground state thus

obtained has $M_{\downarrow}=M_{\uparrow}=\frac{N}{2}$ (as was said, $\psi$ will also have no classical expectation value), and $J_{\downarrow}=J_{\uparrow}=J$.

To obtain energies above the ground state, we need to allow $\psi$ and $\Pi_{\psi}$ to have non-zero expectation value, and solve for the full set of equations of motion. But allowing $\Pi_{\psi}$ to have a non-zero expectation value invalidates the expansion (66). We would have to use the full expression (54), and only perform an expansion once we have the classical values for the fields. That expansion would then truncate by use of the theorems found in [21]. These theorems allow us to determine the energy contribution of the spin-interaction term in the hamiltonian, in particular proving that the higher spin states do not contribute.

To study the theory perturbatively, as long as we consider that $\Pi_{\psi}$ does not acquire an expectation value, we can use the results obtained in this section.

\section{$5.2 \quad$ Non-critical Strings}

In applications to both critical and noncritical strings, linearized eigenvalue problems of this kind appear. In recent studies of the AdS/CFT correspondence [55] a similar equation is seen to determine the spectrum of BPS states. The eigenvalue problem itself plays a role in the nontrivial map between the matrix model and AdS space-time.

While the regular harmonic oscillator participates in the $1 / 2$ BPS map, for the $2 \mathrm{~d}$ noncritical string one requires an inverted oscillator potential. So, for the noncritical strings, the classical stationary solution (with $\Pi_{\phi} \equiv 0$ ) is given by $\phi_{0}(x) \propto \sqrt{x^{2}-\mu}$ [2, 11, 6, 45].

In the background of the classical value for the field $\phi$, we will now want to study the interaction part of the hamiltonian:

$$
\begin{aligned}
\left.\Delta H\right|_{\phi_{0}} & =-\frac{\beta}{4} \int d x f d y \frac{\left[\phi_{0}(x) \psi(y)-\psi(x) \phi_{0}(y)\right]}{\sin ^{2}\left(\frac{x-y}{2}\right)} \Pi_{\psi}(x) \\
& =\int d x f d y \psi(x) K(x, y) \Pi_{\psi}(x),
\end{aligned}
$$

where we have

$$
\int d x \psi(x) \int d y K(x, y) \Pi_{\psi}(x)=-\frac{\beta}{4} \int d x \psi(x) \int d y \phi_{0}(y) \frac{\Pi_{\psi}(y)-\Pi_{\psi}(x)}{\sin ^{2}\left(\frac{x-y}{2}\right)} .
$$

which acts on wave functionals ${ }^{2} \Phi=\int d z f(z) \psi(z)$. This $-K(x, y)$ is the Marchesini-Onofri

\footnotetext{
${ }^{2}$ Recall that the fields $\phi_{\sigma}$ have to obey the following normalization

$$
\int d x \phi_{\sigma}(x)=M_{\sigma} \quad \text { and } \quad \sum_{\sigma} \mathrm{M}_{\sigma}=\mathrm{N}
$$
}


kernel [20, 6]. We conclude that the Marchesini-Onofri kernel comes directly from the spininteraction term of the hamiltonian.

We are interested in solving the eigenvalue problem $(\Delta H) \Phi=\epsilon \Phi$, which can be written in a simpler way. Consider a complete set of eigenfunctions of $K(x, y),\left\{f_{n}(x)\right\}$, with normalization condition

$$
\int d x \phi_{0}(x) f_{n}(x) f_{m}(x)=\delta_{m n} .
$$

Expand the fields $\psi, \Pi_{\psi}$ in this basis:

$$
\Pi_{\psi}(x)=\phi_{0}(x) \sum_{n} f_{n}(x) \Pi_{n} \quad ; \quad \psi(x)=\sum_{n} f_{n}(x) \psi_{n} .
$$

With these expansions, we can write $\Delta H$ as

$$
\Delta H=\sum_{m, n} \psi_{n} \Pi_{m} \int d x f_{n}(x) f d y K(x, y) \phi_{0}(y) f_{m}(y)=\sum_{n} \omega_{n} \psi_{n} \Pi_{n},
$$

where the frequencies $\omega_{n}$ are defined by the following eigenvalue equation

$$
f d y \phi_{0}(y) K(x, y) f_{m}(y)=\omega_{m} \phi_{0}(x) f_{m}(x)
$$

One can use the semiclassical results found in [6] to evaluate these frequencies, as the eigenvalue problems found in each case are equivalent. The result is then:

$$
\omega_{n}=\epsilon_{n}=(n+1) \omega(\mu)+\eta(\mu)
$$

with $\omega(\mu)=\frac{1}{\eta(\mu)}=\frac{2 \beta}{|\ln \mu|}$. The term $\omega(\mu)$ measures the splitting in the singlet energies. These splittings tend to zero in the $\mu \rightarrow 0$ limit (double scaling limit). The term $\eta(\mu)$ gives the difference in energy between the singlet state (corresponding to trivial representation, if we set the interaction term to zero) and the adjoint state due to the interaction term. Up to leading order, $\eta(\mu)$ can be identified with the ground state energy $\omega_{0}$, divergent in the double scaling limit [6].

In [9] the same eigenvalue problem was studied, and it was shown to be equivalent to

$$
\hat{\epsilon} h(\tau)=-\frac{1}{\pi} \int_{-\infty}^{\infty} \frac{h\left(\tau^{\prime}\right)}{4 \sinh ^{2}\left(\frac{\tau-\tau^{\prime}}{2}\right)}+\hat{v}(\tau) h(\tau)
$$

with $\hat{v}(\tau)=-\frac{1}{\pi} \frac{\tau}{\tanh \tau}$, and $\hat{\epsilon}=\epsilon+\frac{\ln \mu}{2 \pi}$, where $\epsilon$ is the energy in the original eigenvalue problem, Furthermore, if the fields $\Pi_{\sigma}$ have a classical expectation value of $J_{\sigma}$, in coordinate space we'll have

$$
\int d x \phi_{\sigma}(x) \partial \Pi_{\sigma}(x)=M_{\sigma} J_{\sigma}
$$


$h(\tau)=\phi_{0}(\tau) f(\tau)$, and $\tau$ is the time-of-flight coordinate: $x=\sqrt{2 \mu} \cosh \tau$. In the energy $\hat{\epsilon}$ we subtracted the divergent piece, $\epsilon_{0} \approx \eta(\mu)$, renormalizing the energy.

The comparison to string theory allows us to interpret this divergent piece of the energy. To perform this comparison, valid for large $\tau$, we have to identify $\tau \sim-\varphi$, where $\varphi$ is the Liouville direction [3]. The divergence that leads to the renormalization of the energy is then basically related with the fact that the string is stretching from the Liouville wall to $-\infty$.

Also in [9], a scattering phase (in the background of the inverted oscillator potential) was conjectured to be:

$$
\delta(\hat{\epsilon})=-\int_{-\infty}^{\infty}\left(\frac{\pi \epsilon^{\prime}}{\tanh \pi \epsilon^{\prime}}+\pi \epsilon^{\prime}\right) d \epsilon^{\prime}
$$

as it is in agreement with the scattering phase of the Liouville model, in the string theory approach. This result was seen to come from solving the Marchesini-Onofri eigenvalue problem, first for the asymptotic limits $\hat{\epsilon} \ll 0$ and $\hat{\epsilon} \gg 0$ [9], and later for any energy [56].

\section{Nontrivial Eigenstates and Backgrounds}

Of major interest in studies and applications of matrix models are possibilities of introducing non-trivial backgrounds in the theory. These are in correspondence with nontrivial states of the many body problem. In the case of free fermions these were for example states representing particles and holes and associated solitonic configurations [53, 36]. In the present case of SpinCalogero problem an interesting class of nontrivial exact eigenstates was given by Ha and Haldane in [21]. They constructed a many body wavefunction of the form is given by

$$
\Psi=\Delta^{\beta} \tilde{\Delta} \chi
$$

where

$$
\begin{aligned}
\Delta & =\prod_{i<j}\left(z_{i}-z_{j}\right) \prod_{i} z_{i}^{-\frac{N-1}{2}} \\
\widetilde{\Delta} & =\prod_{i<j}\left(z_{i}-z_{j}\right)^{\delta_{\sigma_{i} \sigma_{j}}} e^{i \frac{\pi}{2} \operatorname{sgn}\left(\sigma_{\mathrm{i}}-\sigma_{\mathrm{j}}\right)}, \\
\chi\left(\phi_{n}\right) & =\prod_{i} z_{i}^{J_{\sigma_{i}}}=e^{i \sum_{j} J_{\sigma_{j}} x_{j}}=e^{i \sum_{\sigma} J_{\sigma} \int d x x \phi^{\sigma}(x)} .
\end{aligned}
$$

and evaluated the corresponding energy.

It is our interest to consider this state in collective field theory. The strategy is to perform a similarity transformation, but now in terms of the new wavefunction

$$
\Psi_{0}=\Delta^{\beta} \widetilde{\Delta}
$$


The resulting effective hamiltonian describes the theory above the nontrivial quantum state. It can be written as a sum of several terms:

$$
\begin{aligned}
\bar{H} & \equiv \underbrace{\Psi_{0}^{-1} H \Psi_{0}}_{\bar{H}_{1}} \\
& =\underbrace{\sum_{i} D_{i}^{2}}_{\bar{H}_{2}}+\underbrace{\beta \sum_{i<j}\left(\theta_{i j}-\theta_{j i}\right)\left(D_{i}-D_{j}\right)}_{\bar{H}_{3}}+\underbrace{2 \sum_{i \neq j} \theta_{i j} \delta_{\sigma_{i} \sigma_{j}} D_{i}}+\bar{H}_{\text {int }}+\bar{H}_{\text {local }}+E,
\end{aligned}
$$

There are two contributions to the ground state energy, one local, $\bar{H}_{\text {local }}$ ), and the other non local, $E$. They can be determined to be:

$$
\begin{aligned}
E & =E_{0}+\frac{1}{2}(\beta+1) \sum_{\sigma} M_{\sigma}\left(M_{\sigma}-1\right)+\frac{1}{3} \sum_{\sigma} M_{\sigma}\left(M_{\sigma}-1\right)\left(M_{\sigma}-2\right), \\
\bar{H}_{\text {local }} & =\beta \sum_{i \neq j \neq k} \frac{\left(z_{i}+z_{j}\right) z_{i} \delta_{\sigma_{i} \sigma_{k}}}{\left(z_{i}-z_{j}\right)\left(z_{i}-z_{k}\right)}+\beta \sum_{i \neq j} \frac{z_{i} z_{j}}{\left(z_{i}-z_{j}\right)^{2}}\left(2 \delta_{\sigma_{i} \sigma_{j}}-1\right),
\end{aligned}
$$

where the conserved quantity $M_{\sigma}$ (the number of particles with spin $\sigma$ ) is defined as before:

$$
M_{\sigma}=\sum_{i} \delta_{\sigma \sigma_{i}}=\int d x \phi_{\sigma}
$$

The interaction term $\bar{H}_{\text {int }}$ can be written as

$$
\begin{aligned}
& \bar{H}_{i n t}=-\beta \sum_{i \neq j} \theta_{i j} \theta_{j i}(-1)^{\delta_{\sigma_{i} \sigma_{j}}} \prod_{k(\neq i, j)}\left(\frac{z_{k}-z_{i}}{z_{k}-z_{j}}\right)^{\delta_{\sigma_{j} \sigma_{k}}-\delta_{\sigma_{i} \sigma_{k}}} P_{i j}
\end{aligned}
$$

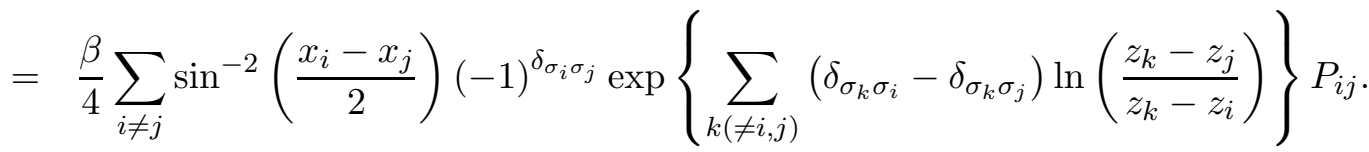

This hamiltonian $\bar{H}$ will now act on bosonic wavefunctions $\chi$. In particular the spin-exchange operator $P_{i j}$ present in the interaction term will now act simply as a translation operator, as was seen in earlier sections.

Comparing (13) with (77), one can easily see that in order for the wavefuncion $\chi$ to be bosonic, and the original hamiltonian fermionic, in both cases $(\tilde{H}$ and $\bar{H}) \Delta^{\beta}$ has to be bosonic. This is due to the factor $\widetilde{\Delta}$ being antisymmetric in exchange of pairs $\left(z_{i}, \sigma_{i}\right)$. Note that for $\beta$ even, the factor $\Delta^{\beta}$ is symmetric under exchange of $z_{i} \leftrightarrow z_{j}$ (and antisymmetric for $\beta$ odd), while the factor $\widetilde{\Delta}$ is always antisymmetric under exchange of pairs $\left(z_{i}, \sigma_{i}\right)$.

As it was done to $H_{\beta+1}$ in section 4 we can obtain the collective form of this hamiltonian (as it is now bosonic) in coordinate space. Writing each term separately, $\bar{H}_{1}$ and $\bar{H}_{2}$ are the same 
as before:

$$
\begin{aligned}
\bar{H}_{1}= & -\sum_{\sigma} \int d x \phi_{\sigma}(x) \partial^{2} \Pi^{\sigma}(x)-\sum_{\sigma} \int d x \phi_{\sigma}(x) \partial \Pi^{\sigma}(x) \partial \Pi^{\sigma}(x) \\
\bar{H}_{2}= & \beta \int d x \phi_{\sigma}(x) \partial^{2} \Pi^{\sigma}(x)- \\
& \quad-\frac{\beta}{2} \sum_{\sigma, \sigma^{\prime}} \int d x \phi_{\sigma}(x) \int d y \phi_{\sigma^{\prime}}(y) \cot \left(\frac{x-y}{2}\right)\left[\partial \Pi^{\sigma}(x)-\partial \Pi^{\sigma^{\prime}}(y)\right] .
\end{aligned}
$$

On the other hand we have a new term $\bar{H}_{3}$, which will be given by:

$$
\bar{H}_{3}=-i \sum_{\sigma} \int d x \int d y \phi_{\sigma}(x)\left[\phi_{\sigma}(y)-\delta(x-y)\right]\left(1-i \cot \left(\frac{x-y}{2}\right)\right) \partial \Pi^{\sigma}(x) .
$$

Finally, we have to deal with the interaction term $\bar{H}_{\text {int }}$ and the local term $\bar{H}_{\text {local }}$. In fact, once in the collective form, $\bar{H}_{\text {local }}$ can be separated into $\bar{H}_{\text {local }}=\sum_{\sigma} H_{\text {local }}^{\sigma}+\sum_{\sigma \neq \sigma^{\prime}} H_{\text {local }}^{\sigma \sigma^{\prime}}$, where:

$$
\begin{aligned}
H_{\text {local }}^{\sigma}= & -\frac{\beta}{3} M_{\sigma}\left(M_{\sigma}-1\right)\left(M_{\sigma}-2\right)+\frac{\beta}{2}(N-2) M_{\sigma}\left(M_{\sigma}-1\right) \\
H_{\text {local }}^{\sigma \sigma^{\prime}}= & 2 \beta \int d x \int d y \int d z \frac{e^{i y}}{e^{i x}-e^{i y}} \frac{e^{i x}}{e^{i x}-e^{i z}} \phi_{\sigma}(x) \phi_{\sigma^{\prime}}(y)\left[\phi_{\sigma}(z)-\delta(x-z)\right] \\
& +\frac{\beta}{4} \int d x \int d y \phi_{\sigma}(x) \sin ^{-2}\left(\frac{x-y}{2}\right) \phi_{\sigma^{\prime}}(y)
\end{aligned}
$$

We conclude that the first term in $\bar{H}_{\text {local }}$ is in fact non local, and contributes directly to the energy.

By knowing that $P_{i j}$ is applied to a bosonic wavefunction, the collective form of $\bar{H}_{\text {int }}=$ $\sum_{\sigma \neq \sigma^{\prime}} H_{i n t}^{\sigma \sigma^{\prime}}$, comes out to be

$$
\begin{aligned}
H_{\text {int }}^{\sigma \sigma^{\prime}}= & -\frac{\beta}{4} \int d x \int d y \frac{\phi_{\sigma}(x) \phi_{\sigma^{\prime}}(y)}{\sin ^{2}\left(\frac{x-y}{2}\right)} \exp \left\{\int d z \ln \left(\frac{e^{i z}-e^{i y}}{e^{i z}-e^{i x}}\right)\left(\phi_{\sigma}(z)-\delta(z-x)\right)\right\} \times \\
& \times \exp \left\{\int d z^{\prime} \ln \left(\frac{e^{i z^{\prime}}-e^{i x}}{e^{i z^{\prime}}-e^{i y}}\right)\left(\phi_{\sigma^{\prime}}\left(z^{\prime}\right)-\delta\left(z^{\prime}-y\right)\right)\right\} \exp \left\{\int_{y}^{x} d w \partial\left(\Pi^{\sigma}-\Pi^{\sigma^{\prime}}\right)\right\} .
\end{aligned}
$$

We find that the final collective hamiltonian will be given by

$$
\bar{H}=\bar{H}_{1}+\bar{H}_{2}+\bar{H}_{3}+\sum_{\sigma \neq \sigma^{\prime}}\left(H_{\text {local }}^{\sigma \sigma^{\prime}}+H_{\text {int }}^{\sigma \sigma^{\prime}}\right)+\bar{E},
$$

where

$$
\bar{E}=\beta^{2} \frac{N\left(N^{2}-1\right)}{12}+\frac{1}{2}(\beta+1) \sum_{\sigma} M_{\sigma}\left(M_{\sigma}-1\right)+\frac{1-\beta}{3} \sum_{\sigma} M_{\sigma}\left(M_{\sigma}-1\right)\left(M_{\sigma}-2\right) .
$$


Our objective is to apply the hamiltonian to the bosonic wavefunction $\chi\left(\phi_{\sigma}\right)$. The eigenvalue equation gives $\bar{H}_{\alpha}(\chi)=E_{\alpha} \chi$, where $\alpha$ refers to any of the terms of the hamiltonian. We now determine the various contributions to the energy.

In order to do so we will need the following results:

$$
\begin{aligned}
\Pi_{\sigma}(x) \chi & =\frac{\delta}{\delta \phi_{\sigma}(x)} \exp \left\{i \sum_{\sigma^{\prime}} J_{\sigma^{\prime}} \int d y y \phi^{\sigma^{\prime}}(y)\right\}=i J_{\sigma} x \chi, \\
\Pi_{\sigma^{\prime}}(y) \Pi_{\sigma}(x) \chi & =i J_{\sigma} x \Pi_{\sigma^{\prime}}(y) \chi=-J_{\sigma} J_{\sigma^{\prime}} x y \chi .
\end{aligned}
$$

For the first terms of the hamiltonian, the results for the corresponding energies are straightforward. From $\bar{H}_{1}$ and $\bar{H}_{3}$ terms we have:

$$
E_{1}+E_{3}=\sum_{\sigma} J_{\sigma}^{2} M_{\sigma}+\sum_{\sigma} J_{\sigma} M_{\sigma}\left(M_{\sigma}-1\right) .
$$

The contribution from the $\bar{H}_{2}$ term is

$$
E_{2}=\beta \sum_{\sigma, \sigma^{\prime}} \int d x \int d y \phi_{\sigma}(x) \phi_{\sigma^{\prime}}(y) \frac{e^{i x} J_{\sigma}-e^{i y} J_{\sigma^{\prime}}}{e^{i x}-e^{i y}}-\beta N \sum_{\sigma} J_{\sigma} M_{\sigma}
$$

The local term does not have operators, and contributes to the energy as it is:

$$
E_{\text {local }}=\sum_{\sigma \neq \sigma^{\prime}} H_{\text {local }}^{\sigma \sigma^{\prime}}
$$

According to [21], these local terms will be seen to cancel with local terms coming from $H_{\text {int }}^{\sigma \sigma^{\prime}}$. We now want to calculate the energy corresponding to this last term of the hamiltonian. The corresponding energy (summing over different spins) is given by

$$
\begin{aligned}
E_{i n t}=-\frac{\beta}{2} & \sum_{\sigma>\sigma^{\prime}} \int d x f d y \frac{\phi_{\sigma}(x) \phi_{\sigma^{\prime}}(y)}{\sin ^{2}\left(\frac{x-y}{2}\right)} \exp \left\{\int d z \ln \left(\frac{e^{i z}-e^{i y}}{e^{i z}-e^{i x}}\right)\left(\phi_{\sigma}(z)-\delta(z-x)\right)\right\} \times \\
& \times \exp \left\{\int d z^{\prime} \ln \left(\frac{e^{i z^{\prime}}-e^{i x}}{e^{i z^{\prime}}-e^{i y}}\right)\left(\phi_{\sigma^{\prime}}\left(z^{\prime}\right)-\delta\left(z^{\prime}-y\right)\right)\right\}\left(\frac{e^{i x}}{e^{i y}}\right)^{\left(J_{\sigma}-J_{\sigma^{\prime}}\right)}
\end{aligned}
$$

We are interested in terms up to third order in fields $\phi_{\sigma}$ and $\Pi_{\sigma}$ (so the $J_{\sigma}$ count as a field, as they are related to the classical value of the field $\Pi_{\sigma}$ ). For that we will basically state the ansatz given in 21] and use their theorems. The main goal is not to have local terms in the final energy. First, we choose $M_{\downarrow} \geq M_{\uparrow}$ (no loss of generality). Now to obtain an energy independent of local terms, we also have to have $0 \leq J_{\sigma}-J_{\sigma^{\prime}} \leq M_{\sigma^{\prime}}-M_{\sigma}+1$ for $\sigma>\sigma^{\prime}$, and in this case 
the theorems proved in the same paper will give:

$$
\begin{aligned}
E_{\text {int }}= & -\frac{\beta}{3} \sum_{\sigma>\sigma^{\prime}} M_{\sigma}\left(M_{\sigma}-1\right)\left(3 M_{\sigma^{\prime}}-M_{\sigma}-1\right)-\beta \sum_{\sigma>\sigma^{\prime}} M_{\sigma}\left(M_{\sigma^{\prime}}-M_{\sigma}\right)\left(J_{\sigma}-J_{\sigma^{\prime}}\right) \\
& +\beta \sum_{\sigma>\sigma^{\prime}} M_{\sigma}\left(J_{\sigma}-J_{\sigma^{\prime}}\right)^{2}-E_{2}-E_{\text {local }} .
\end{aligned}
$$

The conclusions we can draw from this result is that if we restrict $0 \leq J_{\sigma}-J_{\sigma^{\prime}} \leq M_{\sigma^{\prime}}-M_{\sigma}+1$

$\left(\sigma>\sigma^{\prime}\right)$, then all contributions from terms of order $O\left(\left(J_{\sigma}-J_{\sigma^{\prime}}\right)^{3}\right)$ and higher vanish. Also the non-local terms will cancel.

The final result for the energy will be, once all terms are gathered

$$
E=\sum_{\sigma} E^{\sigma}+\sum_{\sigma>\sigma^{\prime}} E^{\sigma \sigma^{\prime}}
$$

where

$$
\begin{aligned}
E^{\sigma}= & \beta^{2} \frac{N\left(N^{2}-1\right)}{24}+\frac{1}{2}\{\beta N+(1-\beta)\} M_{\sigma}\left(M_{\sigma}-1\right)+\frac{1-\beta}{3} M_{\sigma}\left(M_{\sigma}-1\right)\left(M_{\sigma}-2\right) \\
& +J_{\sigma} M_{\sigma}\left(M_{\sigma}-1\right)+J_{\sigma}^{2} M_{\sigma}, \\
E^{\sigma \sigma^{\prime}}= & -\frac{\beta}{3} M_{\sigma}\left(M_{\sigma}-1\right)\left(3 M_{\sigma^{\prime}}-M_{\sigma}-1\right)-\beta M_{\sigma}\left(M_{\sigma^{\prime}}-M_{\sigma}\right)\left(J_{\sigma}-J_{\sigma^{\prime}}\right)+\beta M_{\sigma}\left(J_{\sigma}-J_{\sigma^{\prime}}\right)^{2} .
\end{aligned}
$$

These are the the energies due to 1-spin and 2-spin interactions, and are the restriction of the general case found in [21] to the $S U(2)$ case (the sum on $\sigma>\sigma^{\prime}$ is in fact just one term, $\sigma=\uparrow, \sigma^{\prime}=\downarrow$ ). We have seen therefore how the nontrivial many-body energy of [21] is obtained in the continuum, collective field formalism. This example also serves as a demonstration of the method in describing nontrivial semiclassical backgrounds. Here we had a state where both the charge and the spin field exhibited nonzero classical expectation values. We have seen how the collective field theory is formulated in this new background . The associated collective field fluctuations give the physical degrees of freedom in this background.

\section{Conclusions}

We have given a study of continuum, field theoretic techniques of relevance to matrix and manybody problems. These techniques have definite condensed matter application. We have featured in the text various connections to low dimensional strings. It is expected that the methods that we have described will be of continuing relevance in these subjects and will play a role in understanding nonperturbative physics of low dimensional black holes and noncritical strings [57, 58, 59, 60, 61, 62, 63. This review was concerned with details of the simplest $\mathrm{SU}(2)$ theory. The more general case of $\mathrm{SU}(\mathrm{R})$ was given in [12]. As we have mentioned of particular interest 
to string model applications is the large $R=N$ limit. In this limit one has a full presence of 44 algebra. A basic description of this limit is given in 44] based on the large N WZW model. Generally this limit exhibits an infinite number of bosonic fields and coupling of $W_{\infty}$ degrees of freedom to the original collective boson. Theories of this kind were given in 64 and the recent work [65].

The larger Yangian symmetry and $W_{\infty}$ appearing in these theories might be of broader relevance for example to higher dimensional bosonization [66]. There is also the potential of these models to provide a quantum description of 3 or $4 \mathrm{~d}$ noncritical membranes, as was discussed in [44]. Finally, the continuum collective representation (of the fermion droplet) was seen recently to play a central role in the 5 dimensional AdS/CFT correspondence through $1 / 2$ BPS states [67, 68, 69, 70, 55, 71, 72]. It is expected that the continuum field theories of the extended models will also play a major role in the correspondence, in sectors with less supersymmetry. The collective field map provided a bridge between a one-dimensional matrix theory and a 2dimensional string theory so it is expected that the extension of this will give a mapping of the full AdS/CFT correspondence.

\section{Acknowledgement}

This work was supported in part by the Department of Energy under Contract DE-FG0291ER40688, TASK A, and by the fellowship BD/14351/2003 from Fundação para a Ciência

e Tecnologia, Portugal. We would like to express our gratitude to the referee for careful reading of the manuscript and most constructive comments.

\section{References}

\section{References}

[1] Igor R. Klebanov. String theory in two-dimensions. 1991, hep-th/9108019. Lectures given at Spring School on String Theory and Quantum Gravity, Trieste, Italy, Apr 15-23, 1991.

[2] Antal Jevicki. Developments in 2-d string theory. 1993, hep-th/9309115. Lecture presented at the Spring School in string theory, Trieste, Italy, April 1993.

[3] Paul H. Ginsparg and Gregory W. Moore. Lectures on 2-d gravity and 2-d string theory. 1993, hep-th/9304011. Lectures given at TASI summer school, Boulder CO, June 11-19, 1992.

[4] Sergei Alexandrov. Matrix quantum mechanics and two-dimensional string theory in nontrivial backgrounds. 2003, hep-th/0311273. Ph.D. Thesis. 
[5] Sumit R. Das and Antal Jevicki. String field theory and physical interpretation of $\mathrm{d}=1$ strings. Mod. Phys. Lett., A5:1639-1650, 1990.

[6] David J. Gross and Igor R. Klebanov. Vortices and the nonsinglet sector of the $\mathrm{c}=1$ matrix model. Nucl. Phys., B354:459-474, 1991.

[7] Dmitri Boulatov and Vladimir Kazakov. One-dimensional string theory with vortices as the upside down matrix oscillator. Int. J. Mod. Phys., A8:809-852, 1993, hep-th/0012228.

[8] Vladimir Kazakov, Ivan K. Kostov, and David Kutasov. A matrix model for the twodimensional black hole. Nucl. Phys., B622:141-188, 2002, hep-th/0101011.

[9] Juan Maldacena. Long strings in two dimensional string theory and non- singlets in the matrix model. JHEP, 09:078, 2005, hep-th/0503112.

[10] Denis Uglov. Semi-infinite wedges and the conformal limit of the fermionic calogerosutherland model with spin 1/2. Nucl. Phys., B478:401-430, 1996, hep-th/9601170.

[11] Jean Avan and Antal Jevicki. Collective field theory of the matrix-vector models. Nucl. Phys., B469:287-301, 1996, hep-th/9512147.

[12] J. Avan, A. Jevicki, and J. Lee. Yangian-invariant field theory of matrix-vector models. Nucl. Phys., B486:650-672, 1997, hep-th/9607083.

[13] H. Awata, Y. Matsuo, and T. Yamamoto. Collective field description of spin calogerosutherland models. J. Phys., A29:3089-3098, 1996, hep-th/9512065.

[14] Denis Bernard. An introduction to yangian symmetries. Int. J. Mod. Phys., B7:3517-3530, 1993, hep-th/9211133.

[15] Olivier Babelon and Denis Bernard. Dressing symmetries. Commun. Math. Phys., 149:279306, 1992, hep-th/9111036.

[16] F. D. M. Haldane, Z. N. C. Ha, J. C. Talstra, D. Bernard, and V. Pasquier. Yangian symmetry of integrable quantum chains with long range interactions and a new description of states in conformal field theory. Phys. Rev. Lett., 69:2021-2025, 1992.

[17] D. Bernard, M. Gaudin, F. D. M. Haldane, and V. Pasquier. Yang-baxter equation in long range interacting system. J. Phys., A26:5219-5236, 1993.

[18] E. Billey, J. Avan, and O. Babelon. Exact yangian symmetry in the classical euler-calogeromoser model. Phys. Lett., A188:263-271, 1994.

[19] Kareljan Schoutens. Yangian symmetry in conformal field theory. Phys. Lett., B331:335-341, 1994, hep-th/9401154. 
[20] G. Marchesini and E. Onofri. Planar limit for su(n) symmetric quantum dynamical systems. J. Math. Phys., 21:1103, 1980.

[21] Z N C Ha and F D M Haldane. On models with inverse-square exchange. Phys. Rev., B46:9359-9368, 1992, cond-mat/9204017.

[22] Ian Affleck. Mesons in the large n collective field method. Nucl. Phys., B185:346, 1981.

[23] Chung-I Tan and Xi-te Zheng. Gauge invariant treatment of qcd in two-dimensions in the large n limit. Phys. Rev., D26:2827, 1982.

[24] I. Andric, A. Jevicki, and H. Levine. On the large n limit in sympletic matrix models. Nucl. Phys., B215:307, 1983.

[25] J. Gibbons and T. Hermsen. A generalisation of the calogero-moser system. Physica, D11:337-348, 1984.

[26] S. Wojciechowski. An integrable marriage of the euler equations with the calogero-moser system. Phys. Lett., A111:101-103, 1985.

[27] Alexios P. Polychronakos. Exchange operator formalism for integrable systems of particles. Phys. Rev. Lett., 69:703-705, 1992, hep-th/9202057.

[28] Atish Dabholkar. Fermions and nonperturbative supersymmetry breaking in the onedimensional superstring. Nucl. Phys., B368:293-310, 1992.

[29] Gabriele Ferretti. The untruncated marinari-parisi superstring. J. Math. Phys., 35:44694476, 1994, hep-th/9310002.

[30] Gabriele Ferretti and S. G. Rajeev. Universal dirac-yang-mills theory. Phys. Lett., B244:265269, 1990.

[31] Joseph A. Minahan. Matrix models and one-dimensional open string theory. Int. J. Mod. Phys., A8:3599-3614, 1993, hep-th/9204013.

[32] Joseph A. Minahan and Alexios P. Polychronakos. Integrable systems for particles with internal degrees of freedom. Phys. Lett., B302:265-270, 1993, hep-th/9206046.

[33] Antal Jevicki and Joao P. Rodrigues. Supersymmetric collective field theory. Phys. Lett., B268:53-58, 1991.

[34] Joao P. Rodrigues and Andre J. van Tonder. Marinari-parisi and supersymmetric collective field theory. Int. J. Mod. Phys., A8:2517-2550, 1993, hep-th/9204061.

[35] I. Andric, V. Bardek, and L. Jonke. Collective field excitations in the calogero model. Fizika, B4:93-110, 1995, hep-th/9409191. 
[36] I. Andric, V. Bardek, and L. Jonke. Multi-vortex solution in the sutherland model. J. Phys., A30:717-724, 1997, hep-th/9605196.

[37] Ivan Andric, Velimir Bardek, and Larisa Jonke. Quantum fluctuations of the chernsimons theory and dynamical dimensional reduction. Phys. Rev., D59:107702, 1999, hepth/9811109.

[38] Diptiman Sen and R. K. Bhaduri. Applications of the collective field theory for the calogerosutherland model. Annals Phys., 260:203-223, 1997, cond-mat/9702152.

[39] L. Feher and B. G. Pusztai. Spin calogero models obtained from dynamical r-matrices and geodesic motion. Nucl. Phys., B734:304-325, 2006, math-ph/0507062.

[40] L. Feher and B. G. Pusztai. Spin calogero models associated with riemannian symmetric spaces of negative curvature. Nucl. Phys., B751:436-458, 2006, math-ph/0604073.

[41] F. Calogero. Exactly solvable one-dimensional many body problems. Nuovo Cim. Lett., $13: 411,1975$.

[42] J. Moser. Three integrable hamiltonian systems connected with isospectral deformations. Adv. Math., 16:197-220, 1975.

[43] Masafumi Fukuma, Hikaru Kawai, and Ryuichi Nakayama. Infinite dimensional grassmannian structure of two- dimensional quantum gravity. Commun. Math. Phys., 143:371-404, 1992.

[44] Antal Jevicki. Matrix models, open strings and quantization of membranes. 1996, hepth/9607187. Talk given at Summer 96 Theory Institute: Topics in Non-Abelian Duality, Argonne, IL, 27 Jun - 12 Jul 1996.

[45] Jean Avan and Antal Jevicki. Quantum integrability and exact eigenstates of the collective string field theory. Phys. Lett., B272:17-24, 1991.

[46] Alexios P. Polychronakos. Physics and mathematics of calogero particles. 2006, hepth/0607033.

[47] Michael R. Douglas. Conformal field theory techniques in large n yang-mills theory. 1993, hep-th/9311130. Presented at Cargese Workshop on Strings, Conformal Models and Topological Field Theories, Cargese, France, May 12-26, 1993.

[48] D Senechal. An introduction to bosonization. 1999, cond-mat/9908262.

[49] Joshua Feinberg. Quantized normal matrices: Some exact results and collective field formulation. Nucl. Phys., B705:403-436, 2005, hep-th/0408002. 
[50] Velimir Bardek and Danijel Jurman. 2d calogero model in the collective-field approach. Phys. Lett., A334:98-108, 2005, hep-th/0403194.

[51] A. Jevicki and B. Sakita. The quantum collective field method and its application to the planar limit. Nucl. Phys., B165:511, 1980.

[52] A. Jevicki and B. Sakita. Collective field approach to the large $\mathrm{n}$ limit: Euclidean field theories. Nucl. Phys., B185:89, 1981.

[53] Antal Jevicki. Nonperturbative collective field theory. Nucl. Phys., B376:75-98, 1992.

[54] Alexander G. Abanov and Paul B. Wiegmann. Quantum hydrodynamics, quantum benjamin-ono equation, and calogero model. Phys. Rev. Lett., 95:076402, 2005, condmat/0504041.

[55] Aristomenis Donos, Antal Jevicki, and Joao P. Rodrigues. Matrix model maps in ads/cft. Phys. Rev., D72:125009, 2005, hep-th/0507124.

[56] Lukasz Fidkowski. Solving the eigenvalue problem arising from the adjoint sector of the c $=1$ matrix model. 2005, hep-th/0506132.

[57] Tadashi Takayanagi and Nicolaos Toumbas. A matrix model dual of type 0b string theory in two dimensions. JHEP, 07:064, 2003, hep-th/0307083.

[58] M. R. Douglas et al. A new hat for the $\mathrm{c}=1$ matrix model. 2003, hep-th/0307195. n *Shifman, M. (ed.) et al.: From fields to strings, vol. $3^{*} 1758-1827$.

[59] Joanna L. Karczmarek and Andrew Strominger. Matrix cosmology. JHEP, 04:055, 2004, hep-th/0309138.

[60] John McGreevy, Sameer Murthy, and Herman L. Verlinde. Two-dimensional superstrings and the supersymmetric matrix model. JHEP, 04:015, 2004, hep-th/0308105.

[61] Sumit R. Das, Joshua L. Davis, Finn Larsen, and Partha Mukhopadhyay. Particle production in matrix cosmology. Phys. Rev., D70:044017, 2004, hep-th/0403275.

[62] Joshua L. Davis, Leopoldo A. Pando Zayas, and Diana Vaman. On black hole thermodynamics of 2-d type 0a. JHEP, 03:007, 2004, hep-th/0402152.

[63] Leopoldo A. Pando Zayas and Diana Vaman. Condensing momentum modes in 2-d 0a string theory with flux. 2005, hep-th/0507061.

[64] Jean Avan and Antal Jevicki. Interacting theory of collective and topological fields in twodimensions. Nucl. Phys., B397:672-704, 1993, hep-th/9209036.

[65] Yasuyuki Hatsuda and Yutaka Matsuo. Symmetry and integrability of non-singlet sectors in matrix quantum mechanics. 2006, hep-th/0607052. 
[66] A. Houghton, H. J. Kwon, and J. B. Marston. Multidimensional bosonization. Adv. Phys., 49:141-228, 2000, cond-mat/9810388.

[67] Steve Corley, Antal Jevicki, and Sanjaye Ramgoolam. Exact correlators of giant gravitons from dual n $=4$ sym theory. Adv. Theor. Math. Phys., 5:809-839, 2002, hep-th/0111222.

[68] David Berenstein. A toy model for the ads/cft correspondence. JHEP, 07:018, 2004, hepth/0403110.

[69] Hai Lin, Oleg Lunin, and Juan Maldacena. Bubbling ads space and $1 / 2$ bps geometries. JHEP, 10:025, 2004, hep-th/0409174.

[70] Satoshi Iso, Dimitra Karabali, and B. Sakita. Fermions in the lowest landau level: Bosonization, w(infinity) algebra, droplets, chiral bosons. Phys. Lett., B296:143-150, 1992, hepth/9209003.

[71] Aristomenis Donos and Antal Jevicki. Dynamics of chiral primaries in ads $(3) \mathrm{x} \mathrm{s}^{* *} 3 \mathrm{x} \mathrm{t}^{* *} 4$. Phys. Rev., D73:085010, 2006, hep-th/0512017.

[72] Abhishek Agarwal and Alexios P. Polychronakos. Bps operators in $n=4$ sym: Calogero models and 2d fermions. 2006, hep-th/0602049. 